\title{
p38 Mitogen-activated protein kinase regulates chamber-specific perinatal growth in heart
}

\author{
Tomohiro Yokota, ${ }^{1}$ Jin Li, ${ }^{1}$ Jijun Huang, ${ }^{1}$ Zhaojun Xiong, ${ }^{1,2}$ Qing Zhang, ${ }^{3}$ Tracey Chan, ${ }^{3}$ Yichen Ding,,${ }^{4,5}$ Christoph Rau, ${ }^{1}$ Kevin Sung, ${ }^{4}$ \\ Shuxun Ren, ${ }^{1}$ Rajan Kulkarni, ${ }^{4,6}$ Tzung Hsiai, ${ }^{4,5}$ Xinshu Xiao, ${ }^{3}$ Marlin Touma, ${ }^{7}$ Susumu Minamisawa, ${ }^{8}$ and Yibin Wang ${ }^{1,5}$ \\ ${ }^{1}$ Cardiovascular Research Laboratories, Department of Anesthesiology, Department of Physiology, and Department of Medicine, David Ceffen School of Medicine, UCLA, Los Angeles, California, USA. \\ 2Department of Cardiology, Third Affiliated Hospital of Sun Yat-sen University, Guangzhou, China. Integrative Biology and Physiology, David Geffen School of Medicine, ${ }^{4}$ Department of Bioengineering, \\ School of Engineering and Applied Sciences, ${ }^{5}$ Division of Cardiology and ${ }^{6}$ Division of Dermatology, Department of Medicine, and ${ }^{7}$ Department of Pediatrics, David Geffen School of Medicine, UCLA, Los \\ Angeles, California, USA. ${ }^{8}$ Department of Cell Physiology, Jikei University, Tokyo, Japan.
}

In the mammalian heart, the left ventricle (LV) rapidly becomes more dominant in size and function over the right ventricle (RV) after birth. The molecular regulators responsible for this chamber-specific differential growth are largely unknown. We found that cardiomyocytes in the neonatal mouse RV had lower proliferation, more apoptosis, and a smaller average size compared with the LV. This chamber-specific growth pattern was associated with a selective activation of p38 mitogenactivated protein kinase (MAPK) activity in the RV and simultaneous inactivation in the LV. Cardiomyocyte-specific deletion of both the Mapk14 and Mapk11 genes in mice resulted in loss of p38 MAPK expression and activity in the neonatal heart. Inactivation of p38 activity led to a marked increase in cardiomyocyte proliferation and hypertrophy but diminished cardiomyocyte apoptosis, specifically in the RV. Consequently, the p38-inactivated hearts showed RV-specific enlargement postnatally, progressing to pulmonary hypertension and right heart failure at the adult stage. Chamber-specific p38 activity was associated with differential expression of dual-specific phosphatases (DUSPs) in neonatal hearts, including DUSP26. Unbiased transcriptome analysis revealed that IRE1 $\alpha$ /XBP1-mediated gene regulation contributed to p38 MAPK-dependent regulation of neonatal cardiomyocyte proliferation and binucleation. These findings establish an obligatory role of DUSP/p38/ IRE1 $\alpha$ signaling in cardiomyocytes for chamber-specific growth in the postnatal heart.

\section{Introduction}

In the fetal mammalian heart, when both chambers contribute to a common circulation, the right ventricle (RV) is equivalent in size to the left ventricle (LV) and even dominant over the LV in terms of cardiac output in some species. During the postnatal transition, the LV quickly takes over the RV in terms of contractile function, chamber size, and wall thickness. This differential growth is critical for the 2 ventricles to accommodate the dramatic changes in the circulatory system after birth, including separation of peripheral versus pulmonary circulation, a significant increase in the peripheral resistance for the $\mathrm{LV}$, and a simultaneous decrease in the pulmonary vascular resistance for the RV (1).

The developmental origins of the LV versus the RV are different; the $\mathrm{LV}$ is derived from the first heart field, whereas the RV is derived from the second heart field (2). The differential growth pattern observed between the LV and RV during this postnatal transition period has largely been attributed to cardiomyocyte proliferation and hypertrophy, resulting in a significantly larger LV versus RV in

\section{Related Commentary: p. 5109}

Conflict of interest: The authors have declared that no conflict of interest exists. Copyright: () 2020, American Society for Clinical Investigation.

Submitted: December 23, 2019; Accepted: June 17, 2020; Published: August 31, 2020. Reference information: J Clin Invest. 2020;130(10):5287-5301.

https://doi.org/10.1172/JCl135859. the adolescent and adult heart $(3,4)$. Much of our current understanding of this chamber-specific postnatal growth is based on the prevailing paradigm that it is dictated by external factors such as intrachamber pressure (or sheer stress), hormonal signals, vascular growth, and sympathetic innervation $(5,6)$. However, the intracellular molecular mechanisms and the signal network in cardiomyocytes responsible for the chamber-specific postnatal growth and remodeling are largely unknown.

p38 Mitogen-activated protein kinases (MAPKs) belong to a branch of Ser/Thr protein kinases, with isoforms $\beta, \gamma, \delta$, and $\alpha$ encoded by the genes Mapk11, -12, -13, and -14, respectively. p38 MAPKs were originally identified as stress-induced signaling molecules involved in a variety of cellular stress responses, including inflammation, oxidative injury, mechanical overload, and hormone stimulation $(7,8)$. Extensive evidence from in vivo studies has demonstrated a broad impact of p38 MAPK activity on infection, inflammation, tissue injury, and repair (9). Moreover, recent genetic studies have also demonstrated an important role for specific p38 isoforms in cellular differentiation and growth in skin, muscle, and bone (10-12). In the mammalian heart, p38 activities are implicated in cardiomyocyte hypertrophy, inflammatory gene induction, cardiac fibrosis, cardiomyocyte apoptosis, and suppression of neonatal cardiomyocyte proliferation (13). Although p38 MAPK inhibition has been proposed as a potential therapeutic strategy to treat heart diseases $(14,15)$, cardiomyocyte-specific knockout of p38 $\alpha$ results in an exacerbated form of cardiomyop- 
A

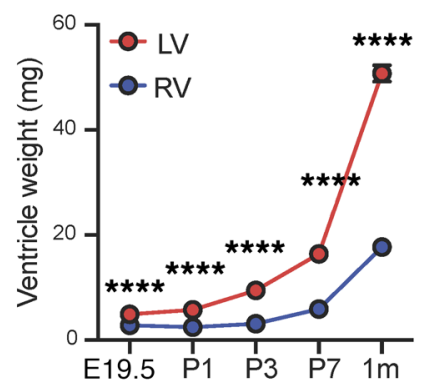

D

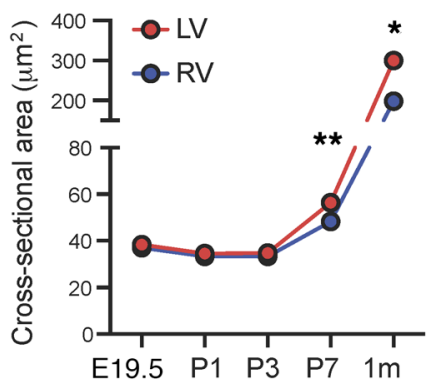

$\mathbf{F}$
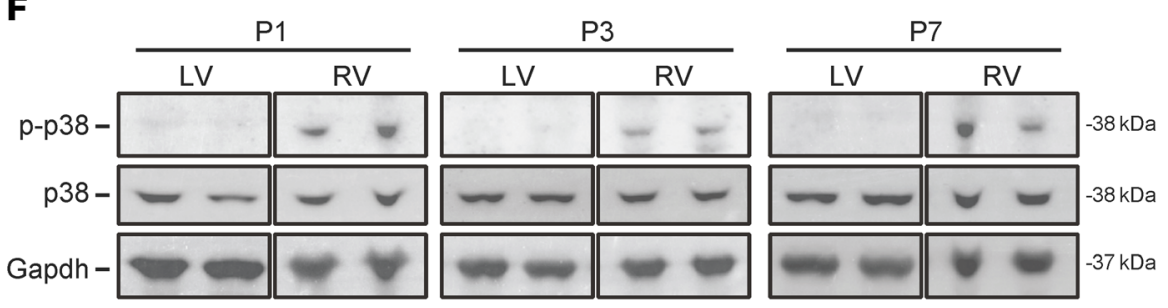

\section{I}
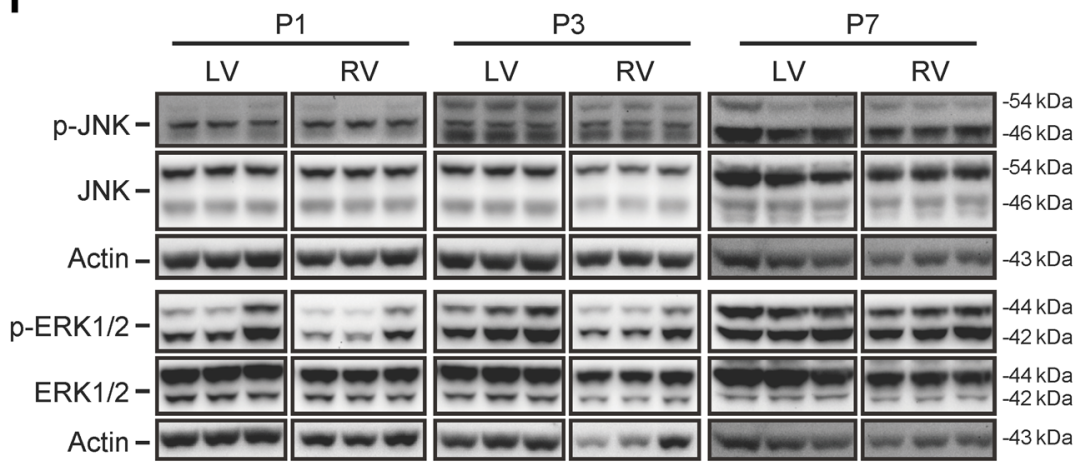

J

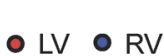

$\mathbf{K}$

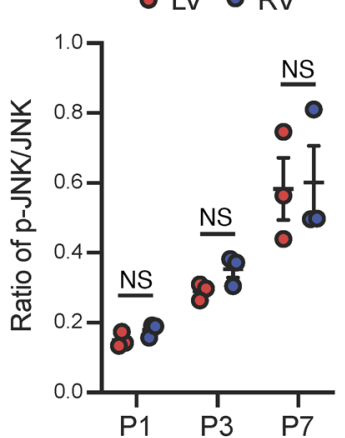

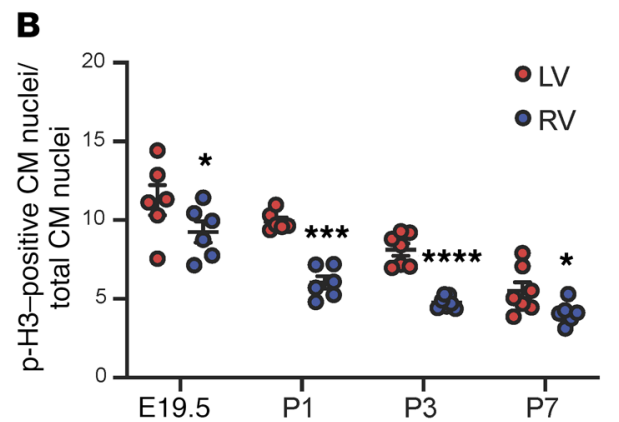

E

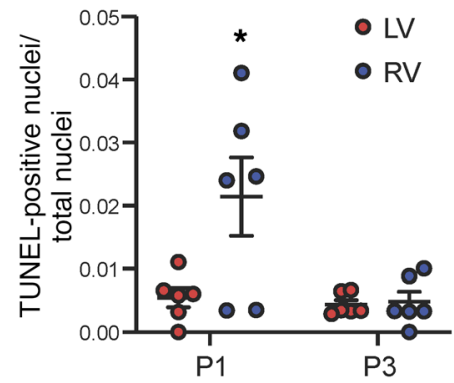

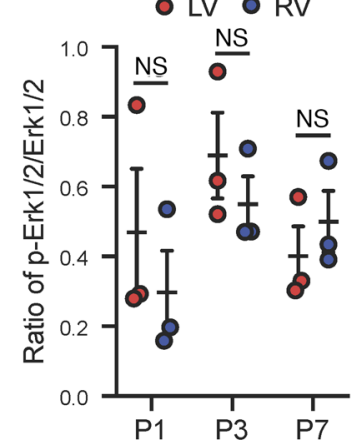

$\mathbf{L}$

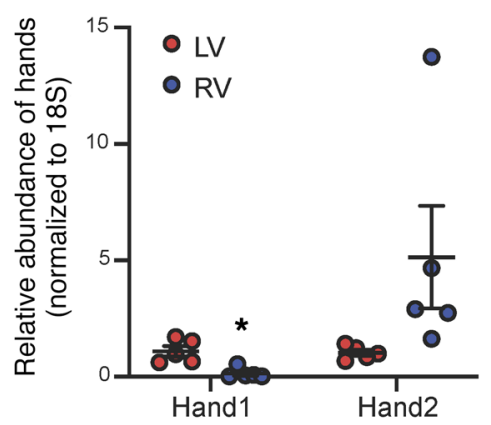

C

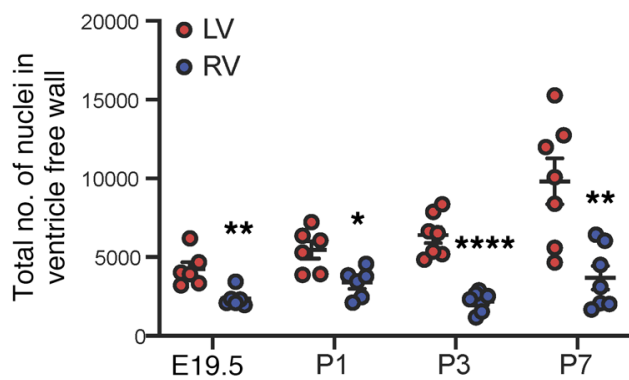

G
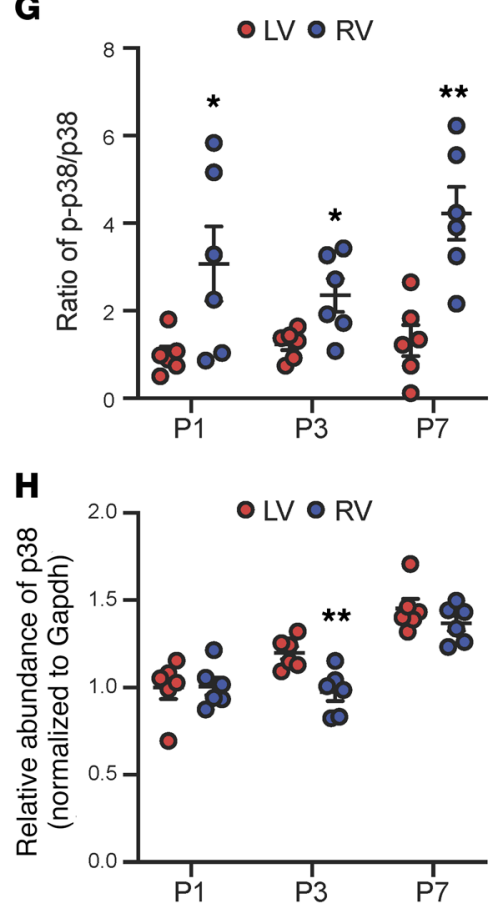
Figure 1. Chamber-specific remodeling and activation of p38 MAPK during postnatal heart development. (A) The tissue weights of the left $(\mathrm{LV})$ and the right (RV) ventricles of mouse perinatal heart at different time points as indicated (E19.5, $n=12 ; \mathrm{P} 1, n=16 ; \mathrm{P3}, n=11 ; \mathrm{P} 7, n=9$; 1 month, $n=9$ ). (B) The phospho-histone H3-positive (p-H3-positive) cardiomyocyte (CM) nuclei/total CM nuclei ratio in the LV vs. RV (E19.5 and $\mathrm{P} 1, n=6 ; \mathrm{P} 3$ and P7, $n=7$ ). (C) Total number of CM nuclei (E19.5 and P1, $n$ $=6 ; P 3$ and P7, $n=7$ ). (D) The cross-sectional area of CMs (E19.5 and P7, $n$ = 6; 1 month, $n=3)$. (E) The number of TUNEL-positive CMs $(n=6)$. (F) Representative immunoblots of phospho-p38, total p38, and Gapdh. (G and $\mathbf{H})$ The phospho-p38 vs. total p38 signal ratio (C) and total p38 (H) $(n=6)$. (I) Representative immunoblots of phosphorylated ERK and JNK vs. total ERK and total JNK in mouse ventricles. (J and $\mathbf{K}$ ) The phosphorylated JNK vs. total JNK signal ratio $(\mathrm{J})$ and phosphorylated ERK vs. total ERK signal ratio (K) $(n=3)$. (L) Chamber specificity of mRNA expression of Hand1 vs. Hand2 detected in the LV vs. RV free wall prepared from P7 neonatal hearts $(n=$ 5). For all panels, data are presented as mean $\pm \mathrm{SEM}$. ${ }^{* * *} P<0.0001,{ }^{* *} P$ $<0.001,{ }^{* *} P<0.01,{ }^{*} P<0.05$ (RV vs. LV). See complete unedited blots in the supplemental material.

athy and pathological remodeling following pressure overload in mice $(16,17)$, highlighting the complexity of the p38 MAPK pathway in both cardiac physiology and pathogenesis of heart failure.

In an effort to uncover the intrinsic signaling mechanisms participating in chamber-specific postnatal growth, we measured the activities of MAPKs in neonatal mouse heart. Among the 3 major MAPK pathways, we found that p38 MAPK activities were significantly and specifically induced in the RV but diminished in the LV during the first week after birth, while the other MAPK pathways including JNK and ERK were not altered. Genetic inactivation of p38 in the neonatal mouse hearts was achieved in a cardiomyocyte-specific Mapk14/11 double-knockout (p38cdKO) mouse model. From histological, cellular, and molecular analyses, we found that the p38-cdKO mice developed remarkable RV-specific enlargement and dilation, which was associated with a significant increase in RV cardiomyocyte proliferation and hypertrophy, and a reduction in apoptosis, while postnatal growth of the LV was largely unaffected. We further demonstrated that differential expression of dualspecific phosphatases (DUSPs), including DUSP26, was correlated with chamber-specific p38 MAPK activities in the neonatal cardiomyocytes. Furthermore, an unexpected IRE1 $\alpha$ / XBP1-dependent signaling was involved in p38 MAPK-dependent regulation of cell cycle gene expression, cardiomyocyte proliferation, and binucleation. Thus, our study has revealed a previously uncharacterized molecular and signaling network necessary for chamber-specific postnatal remodeling and growth in neonatal mouse heart.

\section{Results}

Chamber-specific activation of p38 MAPK during postnatal heart development. In neonatal mouse hearts, we observed a dramatic growth in chamber sizes soon after birth but at different rates between the LVs and RVs (Figure 1A). This was associated with a higher level of cardiomyocyte proliferation in LVs versus RVs, as demonstrated by the number of phospho-histone H3-positive (p-H3-positive) cardiomyocytes and total cardiomyocyte numbers in the LV. In addition, cardiomyocytes in the LV were larger, while the RV showed a transient induction of TUNEL-positive cardiomyocytes (Figure 1, B-E). Because MAPKs are known to be involved in the cellular processes of hypertrophy, proliferation, and programmed cell death, we measured the activation status of 3 major branches of MAPKs, ERK1/2, p38, and JNK, in neonatal hearts (Figure 1, F-K) using free wall tissues from the LV and RV. The chamber origins of the collected tissue were demarcated by the differential expression of Hand1 (for LV) and Hand2 (for RV) (Figure 1L). The activation states of ERK and JNK, as indicated by the phosphorylated versus the total protein levels, did not change during perinatal development nor were they differentially regulated between the LV and RV. In contrast, p38 MAPK showed significant activities in the RV tissue from neonatal hearts during the P1 to P7 period, while it remained inactivated in the LV, below the detection limit, throughout the neonatal period. This chamber-specific activation profile of $\mathrm{p} 38$ MAPK raised the question about its role in the chamber-specific growth pattern in perinatal hearts.

$R V$-specific abnormality in the p38-cdKO mice. To examine the functional role of p38 MAPK activity in neonatal heart, we generated a mouse model (p38-cdKO) with cardiac-specific deletion of both p38 (encoded by Mapk14) and p38 $\beta$ genes (encoded by Mapk11) using Mlc2a-cre-mediated (18) genomic DNA deletion, as described in the Methods. Nearly complete loss of p38 protein expression in the LV and the RV tissues was observed from the p38-cdKO hearts, demonstrating the effectiveness of targeted gene inactivation (Supplemental Figure 1, A and B; supplemental material available online with this article; https://doi. org/10.1172/JCI135859DS1). Among the offspring, the genotype distribution showed an expected ratio of 50:50 between

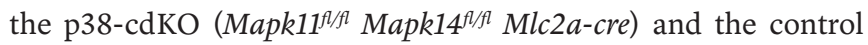

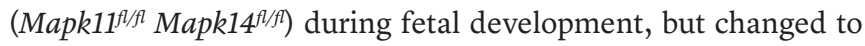
21:79, 35:65, 27:73, and 26:74 at PO, P1, P3, and P7, respectively (Supplemental Table 1), suggesting premature death in the perinatal period. The surviving $\mathrm{p} 38$-cdKO mice showed an abnormal gross morphology of the heart (Figure 2, A and B). Three-dimensional light-sheet microscopy revealed an enlargement of the RV in the p38-cdKO hearts compared with the control (Figure 2, C-F, and Supplemental Videos 1-4). Histological analysis confirmed an increase in RV wall thickness and chamber dimension in the p38-cdKO hearts compared with their littermate controls at P1, P7 (Figure 2, G-J), and beyond (Supplemental Figure 1C). Consistent with these histological observations, the RV weight and the right atrium weight were significantly higher in the p38-cdKO hearts compared with the controls, starting from $\mathrm{P} 1$ onward, but not in the E19.5 fetal hearts (Figure 2, K-M). Remarkably, the p38-cdKO mice showed normal body weight, as well as normal LV, left atrium, and lung weights during the same perinatal period (Figure 2, N-Q). There were no differences in fibrotic area within the myocardium between the 2 genotype groups at P7 and 1 month of age, and no differences between the 2 chambers (Supplemental Figure 1, D and E). Therefore, p38 MAPKs are differentially activated in the perinatal RV, and cardiomyocyte-specific inactivation of p38 MAPKs in neonatal mouse hearts leads to RV-specific enlargement without signs of LV or pulmonary abnormalities.

We also analyzed the dynamic progression of cardiac remodeling in intact mouse hearts based on serial echocardiographic assessment in the postnatal period. To measure the wall thick- 

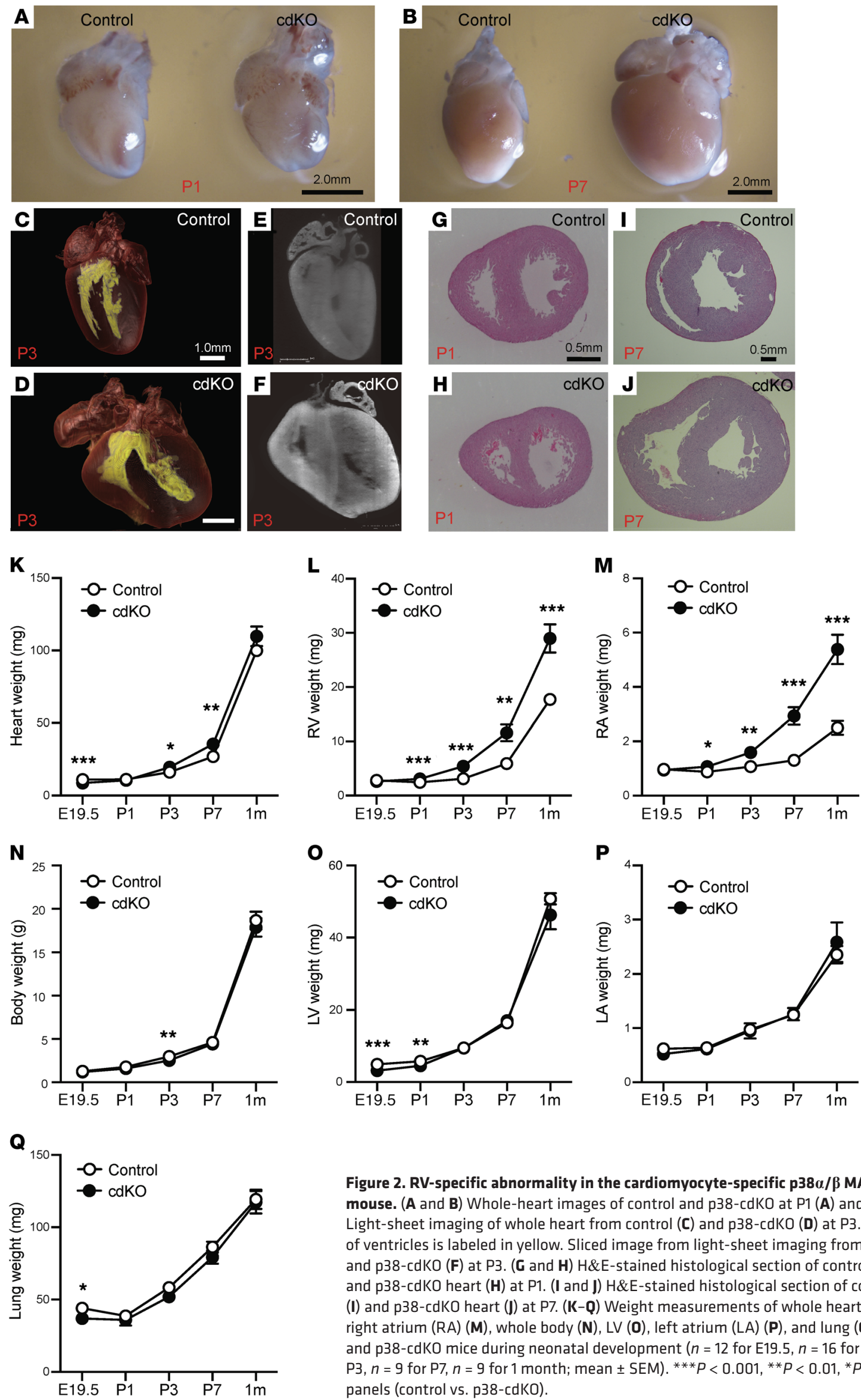

Figure 2. RV-specific abnormality in the cardiomyocyte-specific $p 38 \alpha / \beta$ MAPK-knockout mouse. (A and B) Whole-heart images of control and p38-cdkO at P1 (A) and P7 (B). (C-F) Light-sheet imaging of whole heart from control (C) and p38-cdKO (D) at P3. Inner cavity of ventricles is labeled in yellow. Sliced image from light-sheet imaging from control (E) and p38-cdKO (F) at P3. (G and $\mathbf{H})$ H\&E-stained histological section of control heart (G) and p38-cdKO heart (H) at P1. (I and J) H\&E-stained histological section of control heart (I) and p38-cdKO heart (J) at P7. (K-Q) Weight measurements of whole heart (K), RV (L), right atrium (RA) (M), whole body $(\mathbf{N})$, LV $(\mathbf{O})$, left atrium (LA) $(\mathbf{P})$, and lung $(\mathbf{Q})$ in control and p38-cdKO mice during neonatal development ( $n=12$ for E19.5, $n=16$ for P1, $n=11$ for P3, $n=9$ for P7, $n=9$ for 1 month; mean \pm SEM). ${ }^{* *} P<0.001,{ }^{* *} P<0.01,{ }^{*} P<0.05$ for all panels (control vs. p38-cdKO). 
ness and inner diameter of the LV and RV, we used M-mode echocardiography in the cross-sectional direction, as illustrated in Supplemental Figure 1, F and G, to measure both the LV and RV simultaneously. The p38-cdKO mice showed normal RV wall thickness at P1 but increased significantly from P3 onward (Figure $3 \mathrm{~A}$ ) compared with the controls, while a significant increase in the RV inner diameter was detected starting at P1, indicating an abnormal growth in both thickness and diameter of the RV in the p38-cdKO heart (Figure 3B). There was no change in pulmonary artery velocity at P1 and P3 in the p38-cdKO mice; however, a modest but significant increase in pulmonary artery velocity was observed starting from P7 and progressively elevated beyond the postnatal period for up to 8 months in adult hearts, indicating that pulmonary hypertension developed secondarily to $\mathrm{RV}$ remodeling (Figure $3 \mathrm{C}$ ). Due to the abnormal RV growth, the LV in the p38-cdKO mice was compressed but showed no signs of hypertrophy or dysfunction (Figure 3, D-F). In contrast, a persistent elevation of pulmonary acceleration time was observed, consistent with a state of pulmonary hypertension. However, there were no changes in the pulmonary ejection time in the p38-cdKO hearts up to 8 months of age (Figure 3, G-I). $\mathrm{RV}$ weight increased nearly 2 -fold at 4 and 8 months of age in the p38-cdKO heart versus the control; in contrast, no differences were observed in the LV weight between the 2 genotypes across all time points (Figure 3, J and K). Finally, lung weight significantly increased in the p38-cdKO mouse only after 4 and 8 months of age (Figure 3L). All these data suggested that abnormal RV growth was a primary outcome from p38 inactivation, and pulmonary hypertension was likely a secondary effect. In summary, we establish that cardiomyocyte-specific p38 inactivation leads to RV-specific abnormalities at both morphological and functional levels in the neonatal mouse heart.

p38 MAPK inactivation prolongs cardiomyocyte proliferation specifically in the postnatal $R V$. By immunofluorescent staining, the number of p-H3-positive cardiomyocytes increased significantly in the RV of the p38-cdKO hearts compared with the controls throughout the entire perinatal period, while no significant difference was observed in the LV (Figure 4, A-C). In addition, the total number of cardiomyocytes was significantly higher in the p38-cdKO RV compared with the controls at P3 and P7, while no change was detected in the LV (Figure 4, D and E). Consistent with the observed increase in cardiomyocyte proliferation, the expression of several cell cycle inhibitory genes, p21, Wee1, and $\mathrm{Rb}$, was reduced specifically in the RV of p38-cdKO hearts compared with the controls, whereas no significant differences were detected in the LV (Figure 4, F-I). The phosphorylated Rb $(\mathrm{p}-\mathrm{Rb})$ versus total $\mathrm{Rb}$ ratio was also increased specifically in the p38-cdKO RV at all time points (Figure 4J). We also examined other signaling molecules previously implicated in cardiomyocyte proliferation regulation, including YAP (19-21) and GSK3 $\beta$ (22). They were not affected in either chambers by the p38 inactivation (Supplemental Figure 2, A-C). All of these data suggest that RV-specific p38 activation is a necessary signal to modulate cardiomyocyte proliferation in the RV during normal postnatal development. However, p38-mediated regulation of cardiomyocyte proliferation in the RV does not appear to involve the YAP/ Hippo or GSK3 $\beta$ pathway. p38 MAPK inactivation reduces $R V$ cardiomyocyte apoptosis in the neonatal heart. As shown in Figure 1 and an earlier study in rats (3), RV-specific induction of cardiomyocyte apoptosis may also contribute to chamber-specific differential growth in the postnatal heart. Using a TUNEL assay, we found that the transient induction of cardiomyocyte apoptosis at P1 was significantly blunted in RV by p38 inactivation, whereas no change was observed in the LV (Figure 5, A-C, and Supplemental Figure 3, A and $\mathrm{B}$ ). Consistent with the decrease in apoptosis, the expression of the antiapoptotic protein $\mathrm{Bcl} 2$ was increased in the $\mathrm{RV}$ at $\mathrm{P} 1$ in the p38-cdKO hearts (Figure 5D).

p38 MAPK inactivation induces cardiomyocyte hypertrophy specifically in the neonatal $R V$. By cross-sectional area measurements, cardiomyocytes in the RV showed a significant enlargement in size from P1 to 1 month of age compared with the controls (Figure 5, E-J and M, and Supplemental Figure 3, C-F). On the other hand, the $\mathrm{LV}$ cardiomyocytes showed no differences in size between the p38-cdKO and control hearts (Figure 5, K, L, and N, and Supplemental Figure 3, G-N). These results suggest that RV-specific p38 activation during the postnatal period is necessary to modulate RV cardiomyocyte hypertrophy in the postnatal hearts.

p38 MAPK- and chamber-specific transcriptome programming in the neonatal mouse heart. To uncover the underlying molecular mechanism in RV-specific p38 activation and p38-dependent neonatal heart remodeling, we examined the expression of known upstream regulators of p38 MAPK activities, including MKK3, phospho-MKK3 (p-MKK3), TAB1, and Cdc37 by immunoblot, and detected no differences between the LV and RV (data not shown). To explore further, we conducted transcriptome analysis by RNA sequencing (RNA-seq) using tissues from the LV and RV free wall of P1 and P3 p38-cdKO hearts and the corresponding tissues from the control hearts. Using fold change (FC) greater than 1.2 and $P$ less than 0.05 as selection criteria, we detected 2,242 and 1,789 genes differentially expressed in the RV between the p38-cdKO and control hearts at P1 and P3, respectively. On the other hand, 2,823 and 1,455 genes (same $\mathrm{FC}>1.2$ and $P<0.05$ thresholds) were differentially expressed in the LV from the same cohorts (Supplemental Tables 2-5). We performed principal component analysis (PCA) for all the detected genes (Figure 6A). Withstanding the limitation of the PCA, based on the 2 dimensions with the highest contributions to the variations, we observed a remarkable separation of global gene expression profiles between the LV and RV from P1 to P3 in the control mice, indicating that chamberspecific molecular identity was rapidly established after birth in newborn mouse hearts (Figure 6A). In contrast, the p38-cdKO heart showed a marked shift in global gene expression from their WT counterparts, and the LV and RV transcriptome in the p38cdKO hearts had extensive overlap at P3 (Figure 6A). Therefore, cardiomyocyte-specific p38 MAPK inactivation led to global changes in gene expression in the postnatal hearts, leading to diminished chamber specificity at the transcriptome level.

Based on Gene Ontology (GO) classification of the upregulated genes detected in the p38-cdKO hearts, we found that cell cyclerelated processes were ranked at the top in the RV at both the P1 and P3 time points (Figure 6, B and C, highlighted by brackets), whereas similar functional enrichment was identified in the LV only at P1 but not P3 (Supplemental Figure 4, A and B). Furthermore, GO analysis 
A

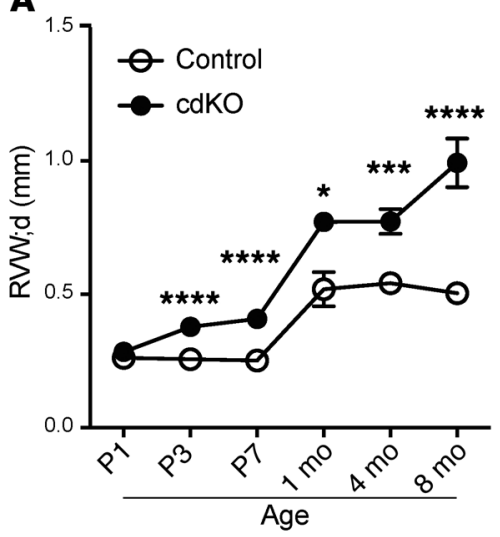

D

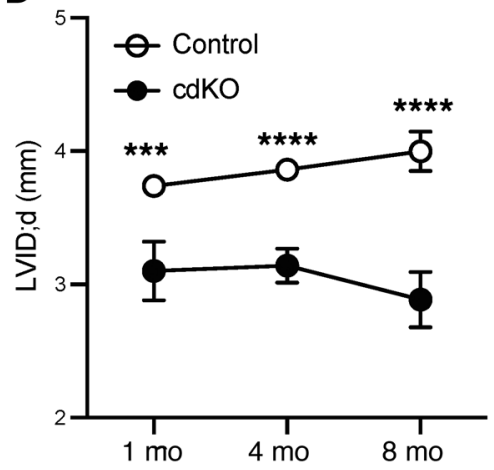

G

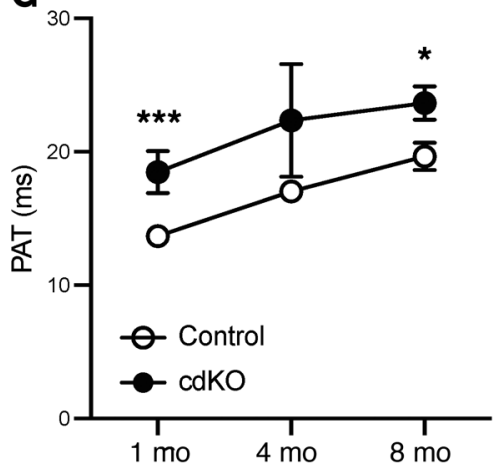

J

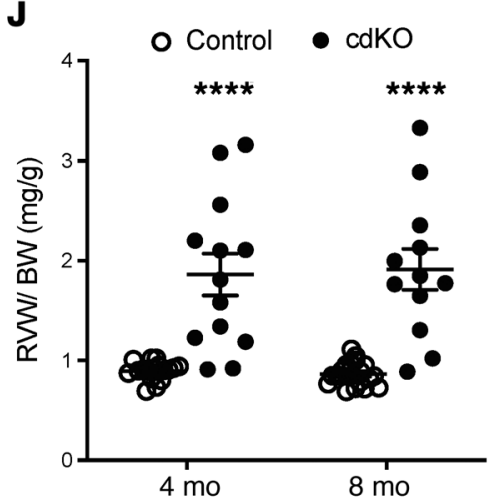

B

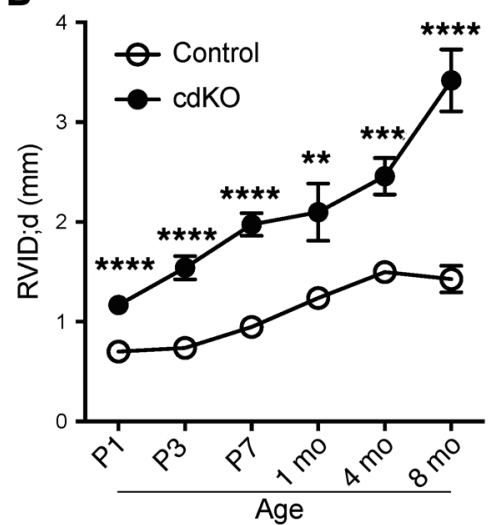

E

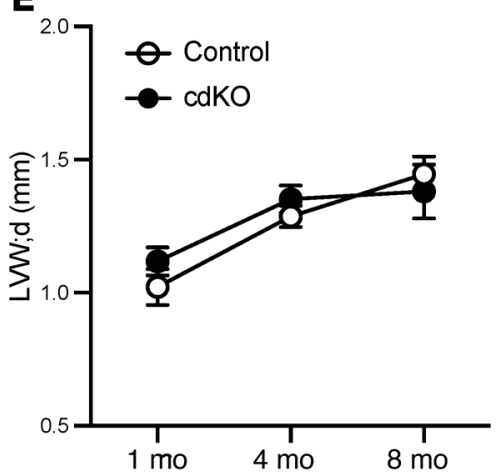

H

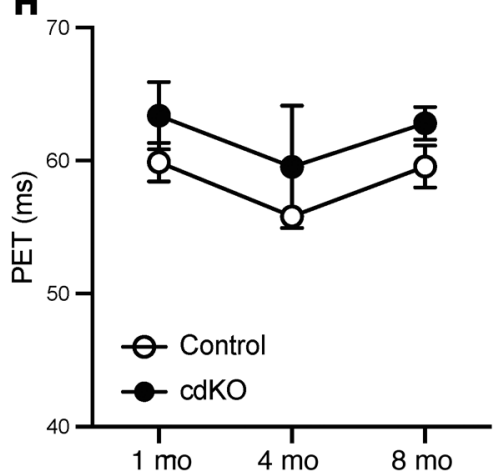

K

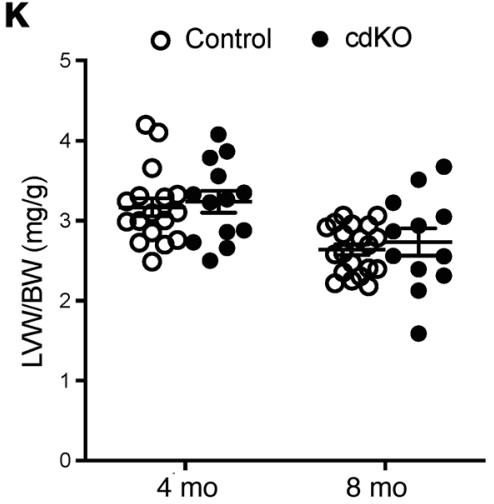

C

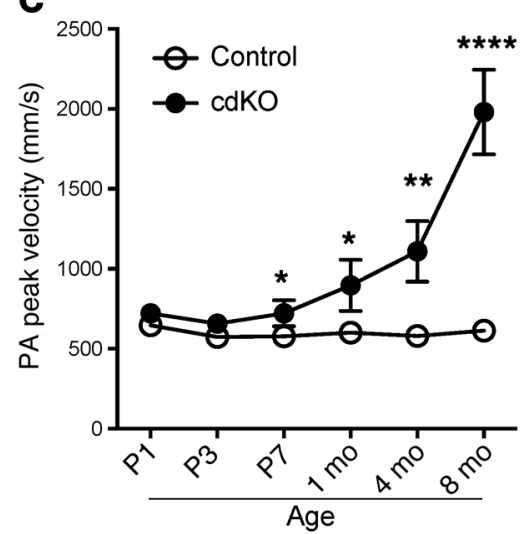

$\mathbf{F}$

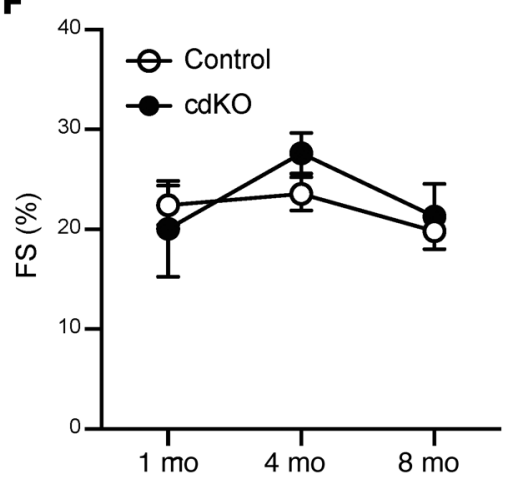

I

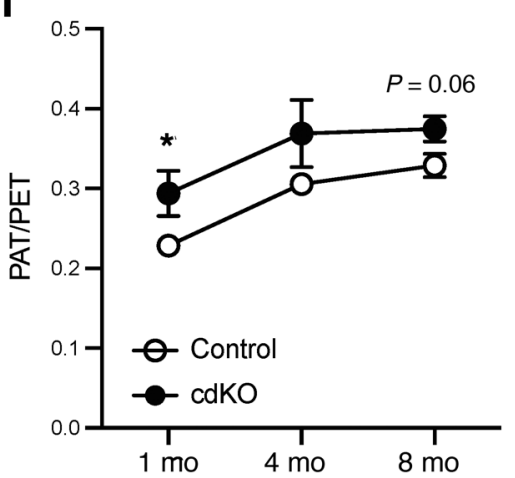

$\mathbf{L}$

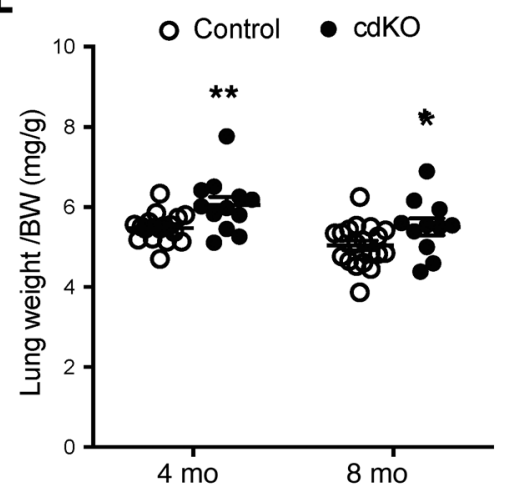


Figure 3. Echocardiogram analysis showing RV-specific abnormalities in the p38-cdKO mouse hearts. (A-C) Serial echocardiographic measurements of (A) end-diastolic RV wall thickness, (B) RV inner diameter, and (C) peak velocity at the pulmonary artery (PA) in the control and p38-cdKO mouse hearts at different perinatal and postnatal time points as indicated (control $n=16$, p38-cdKO $n=12$ at P1 [D1]; control $n=17$, p38-cdKO $n=12$ at P3 [D3]; control $n=20$, p38-cdKO $n=10$ at P7 [D7]; control $n=8$, p38-cdKO $n=5$ at 1 month; control $n=11$, p38-cdkO $n=7$ at 4 months; control $n=$ 10, p38-cdKO $n=6$ at 8 months; mean \pm SEM). (D-F) LV echocardiogram parameters include $(\mathbf{D})$ end-diastolic LV inner chamber diameter, $(\mathbf{E})$ end-diastolic LV wall thickness, and (F) fractional shortening (FS) in the postnatal hearts (control $n=8$, p38-cdKO $n=5$ at 1 month; control $n=14$, p38-cdKO $n=10$ at 4 months; control $n=10$, p38-cdKO $n=6$ at 8 months; mean \pm SEM). (G-I) Pulmonary circulation indicators are (G) pulmonary artery acceleration time (PAT), (H) pulmonary ejection time (PET), and (I) PAT/PET ratio in the postnatal hearts (control $n=8, p 38-c d K O n=5$ at 1 month; control $n=14$, p38-cdKO $n=10$ at 4 months; control $n=10$, p38cdKO $n=6$ at 8 months; mean \pm SEM). (J-L) RV free wall (J), LV free wall (K), and lung (L) weight to body weight ratios at 4 and 8 months (control $n=17$, p38-cdkO $n=13$ at 4 months; control $n=20$, p38-cdKO $n=12$ at 8 months; mean \pm SEM). ${ }^{* * *} P<0.0001,{ }^{* *} P<0.001,{ }^{* *} P<0.01,{ }^{*} P<0.05$ for all panels (control vs. p38-cdKO).

of the downregulated genes in the p38-cdKO heart revealed a significant enrichment for apoptosis in the RV at P1 (Figure 6, D and $\mathrm{E}$, highlighted by an arrow), whereas no such signal was detected in the LV (Supplemental Figure 4, C and D). Further analysis of the differentially expressed genes revealed a significant overlap between the LV and RV in the genes affected by $\mathrm{p} 38$ inactivation (Figure $6 \mathrm{~F}$ ). The vast majority of the genes affected by $\mathrm{p} 38$ inactivation showed modest FCs (Supplemental Figure 4E and Figure 6G). Although the cell cycle-related genes were ranked at the top based on FCs in both the LV and RV, the magnitudes of p38-dependent gene expression changes were much greater in the RV than in the LV (Figure 6H). This was consistent with the differential p38 activities observed in the 2 chambers, further supporting the notion that cell cycle regulation in the neonatal heart is p38 activity dependent. Among the differentially expressed genes between the $\mathrm{LV}$ and $\mathrm{RV}$, there were several known DUSPs that could target p38 (Supplemental Table 6). In particular, DUSP26 showed higher expression in the RV versus the $\mathrm{LV}$ at both $\mathrm{P} 3$ and $\mathrm{P}$, as validated by reverse transcription PCR (RT-PCR) (Figure 7A). To test if differential expression of DUSP26 contributed to 38 MAPK-mediated signaling, we knocked down DUSP26 in rat neonatal ventricular myocytes (NRVMs) (Supplemental Figure 4F), which indeed led to a significant activation of p38 activity in the NRVMs (Figure 7B). Interestingly, DUSP26 inactivation also enhanced the expression of a number of RV-enriched genes but reduced the expression of a number of $\mathrm{LV}$-enriched genes (Figure 7C), supporting its regulatory role in chamber specificity. Furthermore, DUSP26 inhibition reduced the basal proliferative activity of NRVMs and this effect was completely blunted by p38 MAPK inhibition (Figure 7D), suggesting again that LV-specific p38 inactivation is a downstream event of DUSP26 and DUSP26/p38 MAPK signaling contributes to chamber-specific neonatal cardiomyocyte proliferation.

XBP1 is a downstream molecule mediating $p 38$ MAPK-regulated cardiomyocyte proliferation. In order to establish the molecular basis for the observed p38-dependent regulation of cell cyclerelated genes, we performed whole-genome rVISTA analysis (https://rvista.dcode.org/) for the genes affected by p38 inactivation. A number of transcription factors were identified as potential upstream regulators in the RV at P1 or P3, including E2F, which is a well-established master regulator of the cell cycle (Figure 8A). Unexpectedly, however, we also found XBP1, an ER stress-response factor, among the top candidate transcription factors for the p38-regulated genes in the RV at both the $\mathrm{P} 1$ and $\mathrm{P} 3$ time points and in the $\mathrm{LV}$ at P1 (Figure $8 \mathrm{~A}$ and Supplemental Figure 5).

IRE1 $\alpha / X B P 1$ in the regulation of neonatal cardiomyocyte proliferation. To validate if XBP1 activity was indeed related to p38mediated regulation in neonatal hearts, we examined the expression of IRE1 $\alpha$, an upstream activator of XBP1 (23), and found that is was also significantly upregulated in the p38-cdKO RVs (Figure 8B), while the expression of another ER stress regulator, ATF6, was not affected by $\mathrm{p} 38$ inactivation in the RV (Supplemental Figure 6, $\mathrm{A}$ and B). In the DUSP26-knockdown cardiomyocytes, IRE1 $\alpha$ expression was also significantly reduced (Figure 7C). During ER stress, IRE1 $\alpha$-mediated Xbp1 mRNA splicing results in the production of nucleus-localized spliced XBP1 (sXBP1) protein for downstream gene expression $(24,25)$. sXBP1 was detected at a lower level in the RV than in the LV in the control neonatal hearts, correlating well with the differential p38 activities between the 2 chambers. However, sXBP1 was significantly upregulated only in the RV of the p38-cdKO hearts (Figure 8C), with a concurrent reduction in the cytoplasmic sXBP1 (Supplemental Figure 6C). These data indicate that $\mathrm{p} 38$ MAPK inactivation induces XBP1 activity via IRE1 $\alpha$-mediated posttranscriptional splicing and nuclear translocation.

To directly demonstrate the cell-autonomous effect of p38 activity on IRE1 $\alpha /$ XBP1 signaling, we treated NRVMs with the p38 MAPK-specific inhibitor SB202190 $(10 \mu \mathrm{M})$, which led to a significant induction of IRE1 $\alpha$ expression (Figure 8D). As expected, the ratio between the active $\mathrm{XBP} 1$ versus the inactive, unspliced XBP1 (uXBP1) was also increased following p38 MAPK inhibition, similar to what was observed in the p38-cdKO heart (Figure 8E). Furthermore, we found that ectopic expression of IRE1 $\alpha$ in NRVMs was sufficient to enhance myocyte proliferation in the same manner as p38 inhibition (Figure 9, A and B, and Supplemental Figure 6D). Interestingly, the presence of binucleated myocytes, a sign of myocyte growth and maturation, was also induced in the NRVMs treated with either p38 inhibitor or IRE1 $\alpha$ expression (Figure 9C).

Finally, we inactivated XBP1 expression using an siRNA (siXbp1) and simultaneously inhibited p38 activity using SB202190 in NRVMs (Supplemental Figure 6E). XBP1 inactivation significantly blunted p38 inhibition-induced NRVM proliferation (Figure 9, D and E, and Supplemental Figure 6F). A cell cycle regulatory gene, Ccnb2 (cyclin B2), was identified as an XBP1-regulated gene based on rVISTA analysis (Supplemental Figure $6 \mathrm{G}$ ), and its expression was upregulated by p38 inhibition and this induction was also blunted by XBP1 inactivation (Figure 9F). Notably, IRE1 $\alpha /$ XBP1 signaling had no effect on neonatal cardiomyocyte hypertrophy (Supplemental Figure 6, H and I). Taken together, these in vivo and in vitro data suggest that IRE1 $\alpha$ / XBP1 signaling is a previously uncharacterized downstream target of p38 activity in neonatal cardiomyocytes, contributing to p38-mediated chamber-specific regulation of cardiomyocyte proliferation and binucleation (26-30). 
A
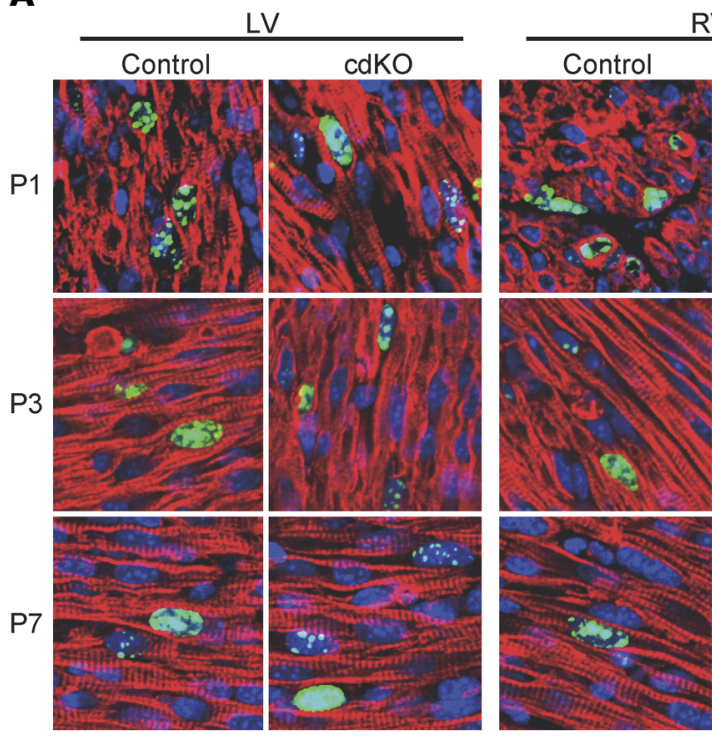

RV

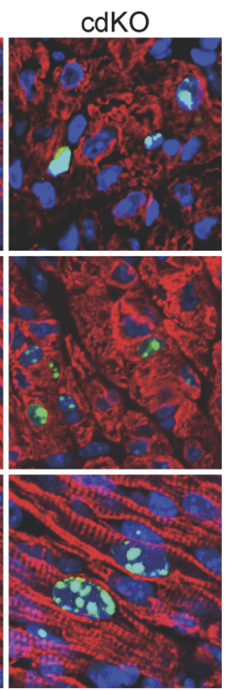

$\mathrm{p}-\mathrm{H} 3 / \mathrm{TPM}$

E

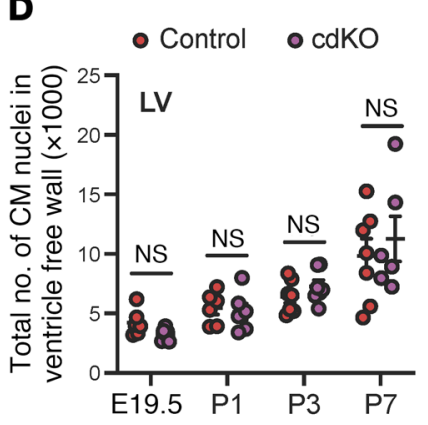

F

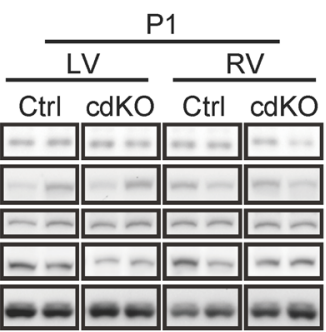

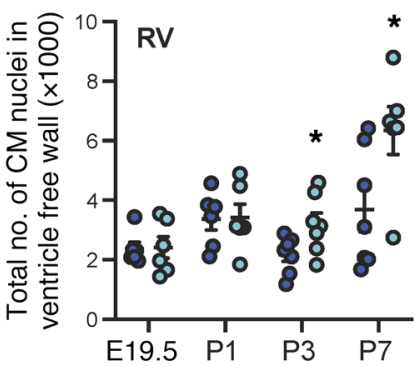

B

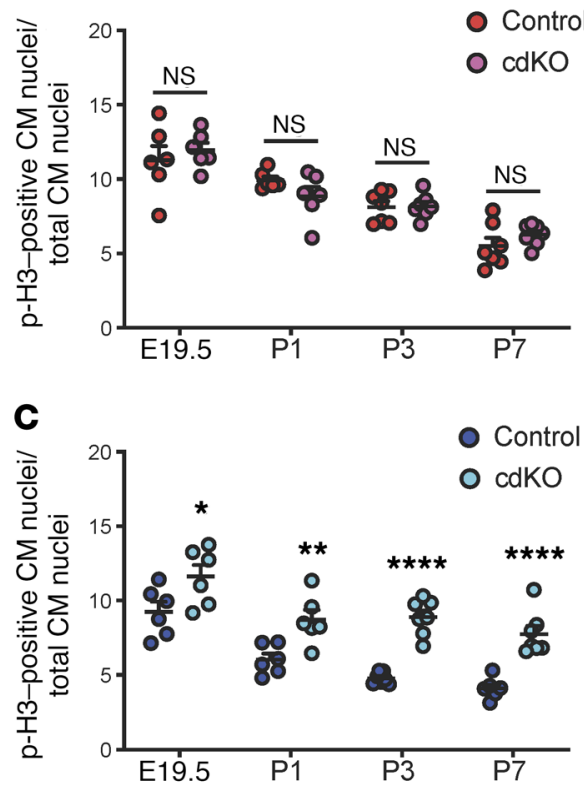

G

- LV control - LV cdKO

- RV control o RV cdKO

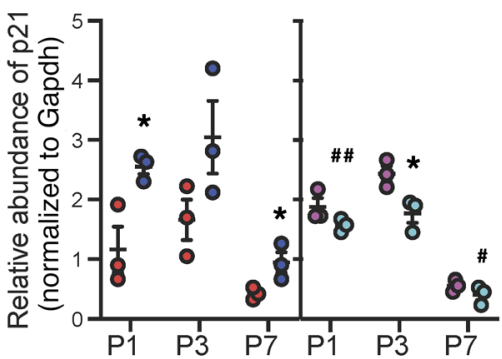

J

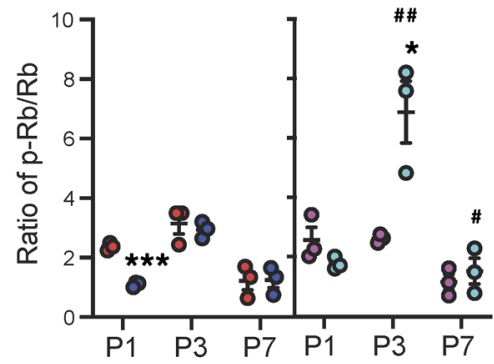

Figure 4. RV-specific impact of p38 MAPK inactivation on cardiomyocyte proliferation in the neonatal heart. (A) Representative images of p-H3/tropomyosin (TPM) staining of control and p38-cdKO hearts at P1, P3, and P7. Original magnification, $\times 120$. (B and C) Quantification of p-H3-positive cardiomyocyte (CM) nuclei/total CM nuclei/section in the LV (B) and RV (C) from control and p38-cdKO during early postnatal development (control and p38-cdKO $n=6$ at E19.5 and P1, $n=7$ at P3 and P7; mean \pm SEM). (D and E) Total number of CM nuclei/section in the neonatal mouse in LV (D) and RV free wall (E) from control and p38-cdKO at different time points (control and p38-cdKO $n=6$ at E19.5 and P1, $n=7$ at P3, control $n=7$ and p38-cdKO $n=6$ at P7; mean \pm SEM). (F-J) Representative immunoblots (F) and quantification of protein expression of p21 (G), Wee1 (H), Rb (I), and p-Rb/Rb ratio (J). ${ }^{* * * * P<0.0001,}$ ${ }^{* * *} P<0.001,{ }^{* *} P<0.01,{ }^{*} P<0.05$ for all panels (LV vs. RV of control or $p 38$-cdKO); ${ }^{\#} P<0.01,{ }^{\#} P<0.05$ (control vs. p38cdKO in RV). 

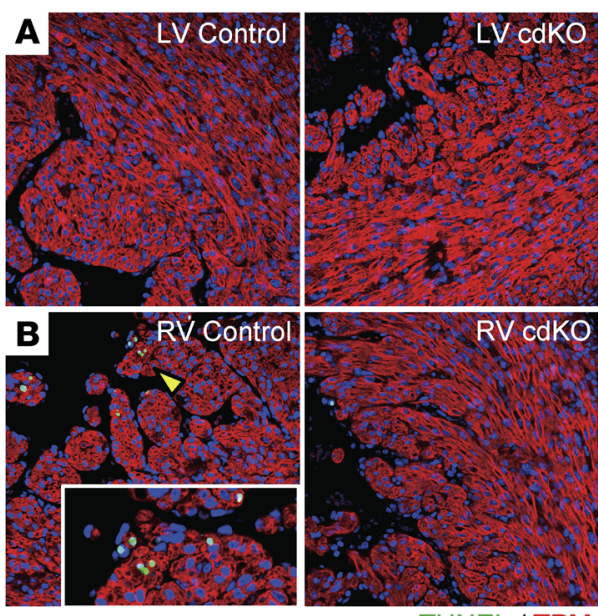

TUNEL / TPM

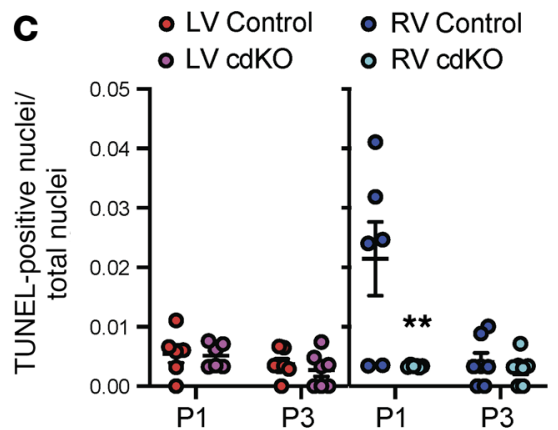

D
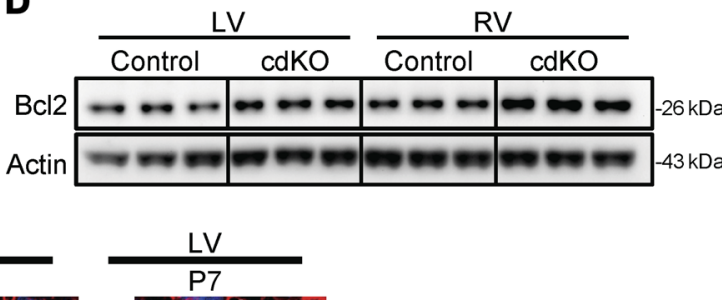
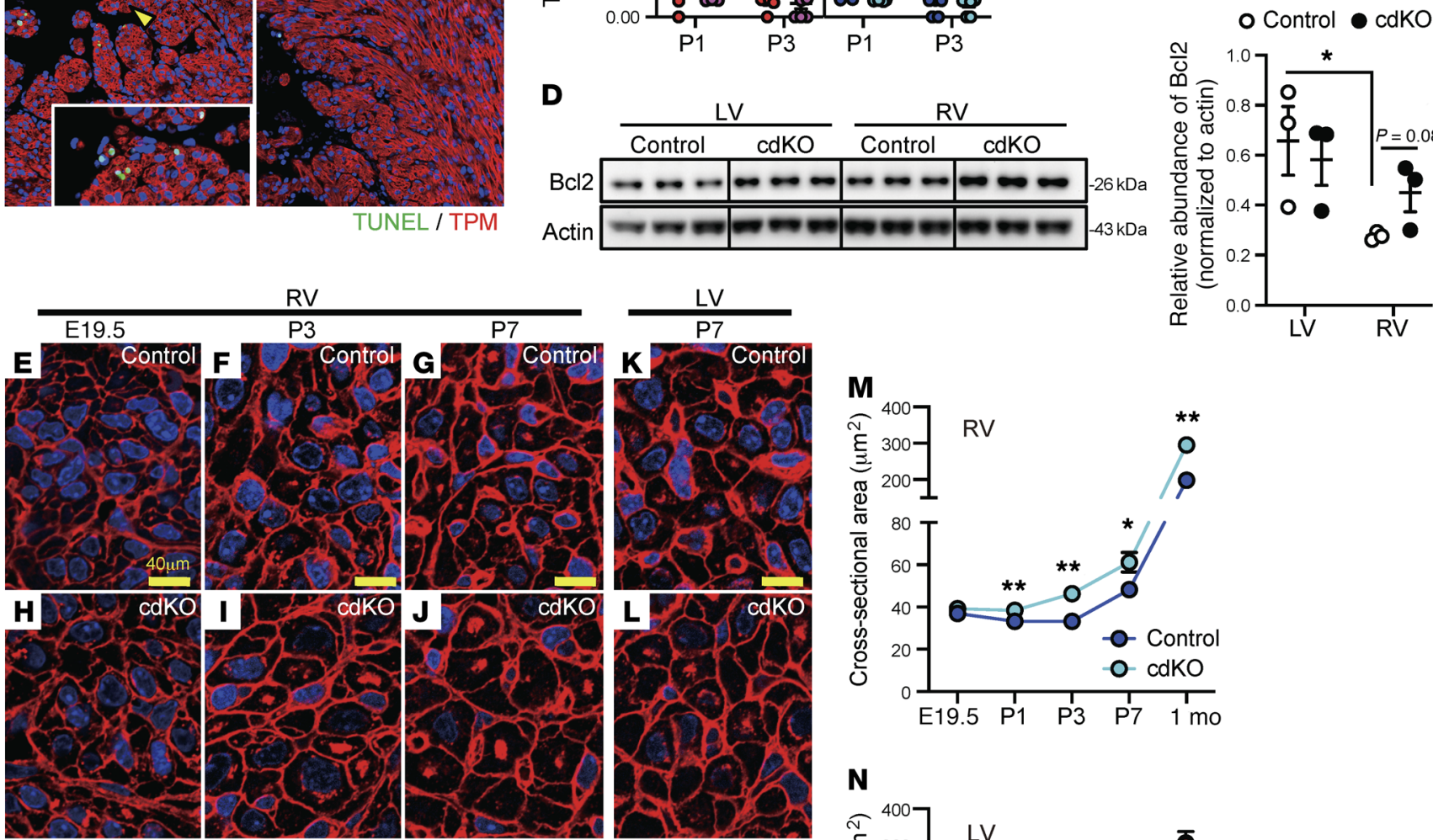

WGA

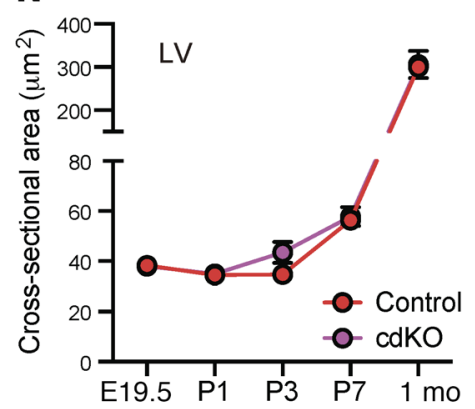

Figure 5. RV-specific effects of p38 MAPK inactivation on cardiomyocyte death and hypertrophy. (A and B) Representative images of TUNEL and tropomyosin (TPM) staining in the LV (A) and RV (B) of the control and p38-cdKO hearts at P1. Original magnification, $\times 20$. Arrowhead indicates area at higher magnification $(\times 60)$ in the inset. (C) Percentage TUNEL-positive cardiomyocyte nuclei/total cardiomyocyte nuclei (control and p38-cdK0 $n=6$ at P1 and $n=7$ at P3; mean \pm SEM). (D) Immunoblot and quantification of the Bcl2 protein level in the neonatal hearts at P1 $(n=3 ;$ mean \pm SEM). (E-L) Representative images of wheat germ agglutinin (WCA) staining in the RV from control or p38-cdKO hearts at E19.5 (E and $\mathbf{H})$, P3 (F and I), and P7 (G and J) time points, and in the LV from control or p38-cdKO hearts at P7 (K and $\mathbf{L})$. Scale bar: $40 \mu \mathrm{m}$. (M and $\mathbf{N})$ Cross-sectional area of cardiomyocytes from the RV (M) and LV (N) during postnatal development (control and p38-cdKO at 1 month $n=3$, other groups $n=6 ;$ mean $\pm S E M)$. ${ }^{* *} P<0.01$, ${ }^{*} P<$ 0.05 for all panels (control vs. p38-cdKO).

\section{Discussion}

In this report, we have uncovered an intrinsic cellular signaling pathway in RV-specific postnatal growth. We found that p38 MAPK activity is selectively and dynamically induced in an RV-specific manner and associated with lower levels of cardiomyocyte hypertrophy, proliferation, and a higher level of apoptosis in the RV versus the LV in perinatal mouse hearts. Car- diomyocyte-specific inactivation of $\mathrm{p} 38 \alpha$ and $-\beta$ isoforms led to RV-specific enlargement in postnatal hearts, likely contributed by elevated proliferation and hypertrophy concomitant with reduced apoptosis in the RV. Mechanistically, we establish that IRE1 $\alpha$ / $\mathrm{XBP} 1$ signaling is a previously uncharacterized downstream pathway of p38 MAPK that contributes to cardiomyocyte proliferation. Therefore, our study has revealed an unexpected role for 2 
A
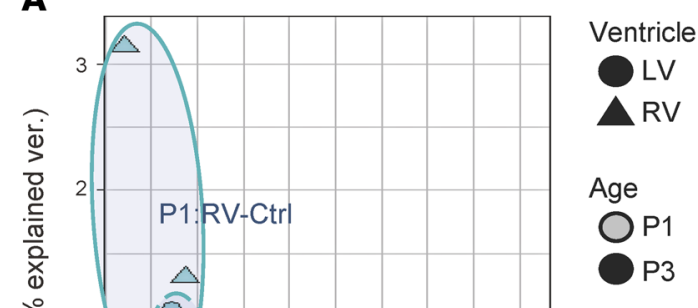

ำ.

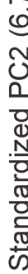

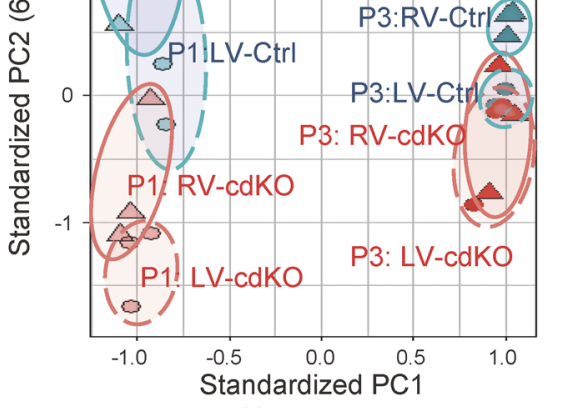

(39.6\% explained ver.)

B

cdKO/Control upregulated

in $\mathrm{RV}$ at $\mathrm{P} 1$

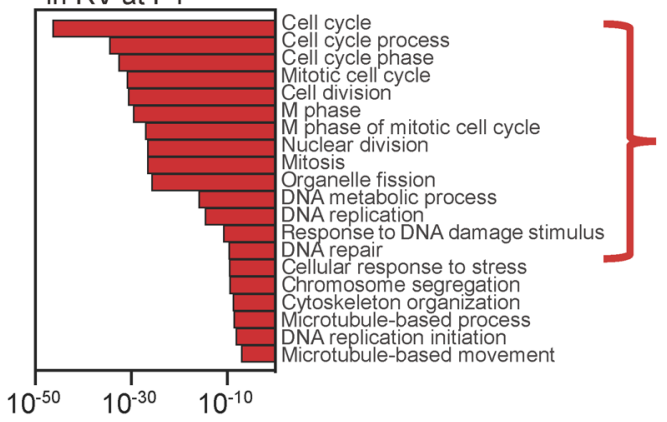

D

cdKO/Control downregulated

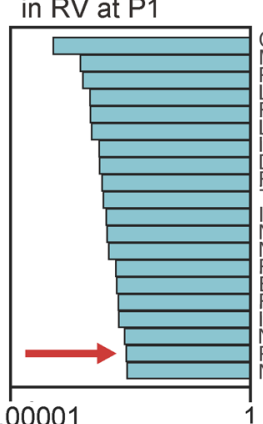

Cell activation

Potassium insport

ymphocyte differentiation

Response to wounding

eukocyte activation

nflammatory respons

Regulation of transcription

Transcription

racellular signaling cascad

gative regulation of gene expression

Genotype

Control

cdko

Figure 6. Transcriptome changes in the p38-cdKO heart. (A)

Principal component analysis (PCA) of all expressed genes in the WT $(n=3)$ and p38-cdKO $(n=3)$ in the LVs and RVs of hearts at $\mathrm{P} 1$ and $\mathrm{P} 3$ time points. Circles represent PCA domains from each experimental group as labeled. (B and C) Gene Ontology (CO) classification of upregulated genes in the p38-cdKO RV compared with control at P1 (B) and P3 (C). (D and E) GO classification of downregulated genes in the p38-cdKO RV compared with control at P1 (D) and P3 (E). (F) Venn diagram showing the overlap of differentially expressed genes (DEGs) in p38-cdKO between the LV and RV at P1 $(n=3)$. ( $\mathbf{G}$ and $\mathbf{H})$ Heatmaps showing fold changes (logFC) of the DEGs shared by both ventricles at P1 ( $n=1,291$ genes) (C) and the cell cycle regulatory genes ( $n=121$ genes) $(\mathbf{H})$.

$\mathbf{F}$

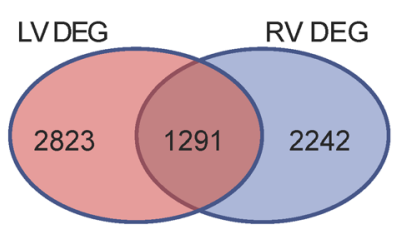

G all genes(1291 genes)

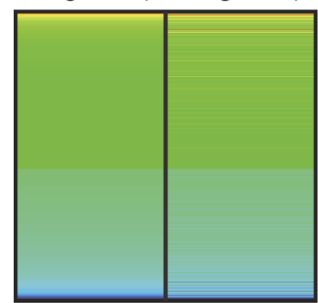

LV

RV

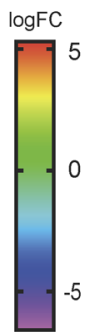

C control upregulated

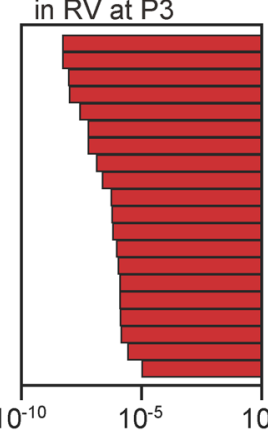

Mitotic cell cycle

(a)

$M$ phase

M phase of mitotic cell cycle Nuclear division

Mitosis

Organelle fission

-based movement

Biological adhesion Cell division

Cycle

Regulation of phosphate metabolic process Regulation of phosphorus metabolic process Metanephros development Regulation of phosphorylation Extracellular structure organization

E

E cdKO/Control downregulated in $\mathrm{RV}$ at $\mathrm{P} 3$

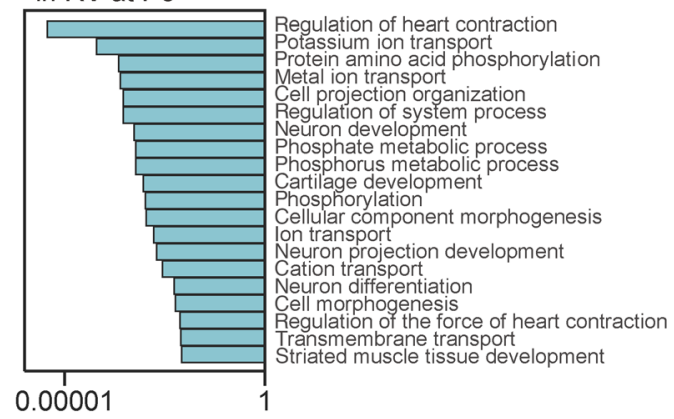

H

ommon DEGs

Cell cycle genes (121 genes) $)_{\log F C}$

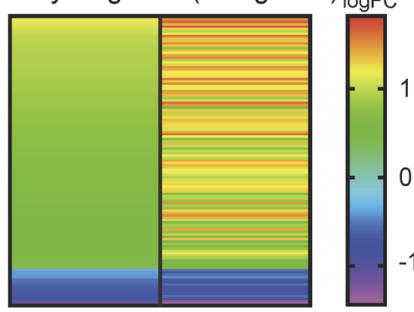

LV

RV 


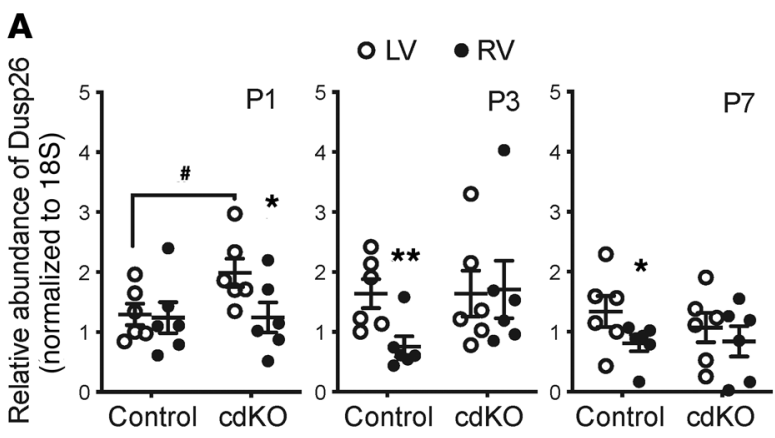

B
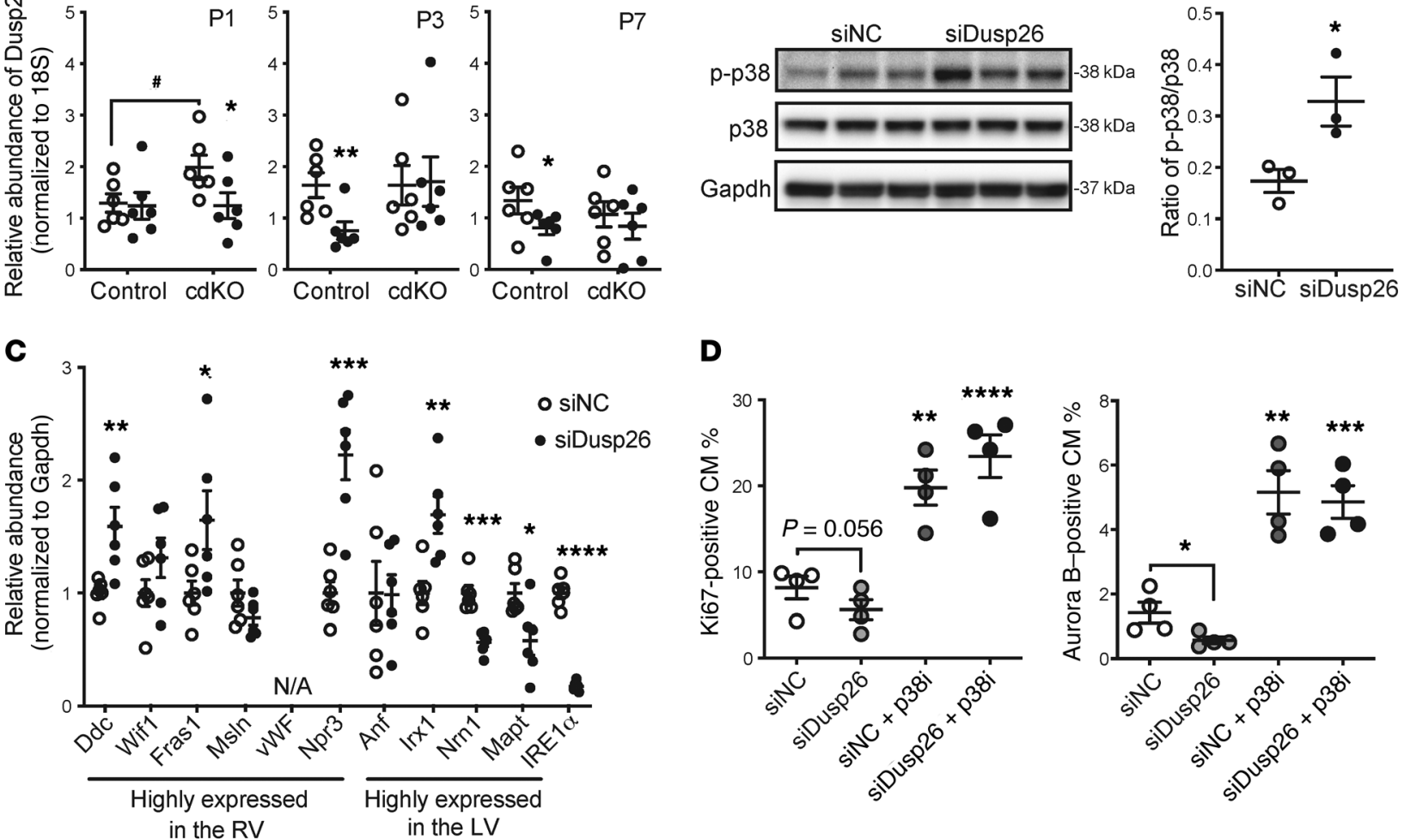

D
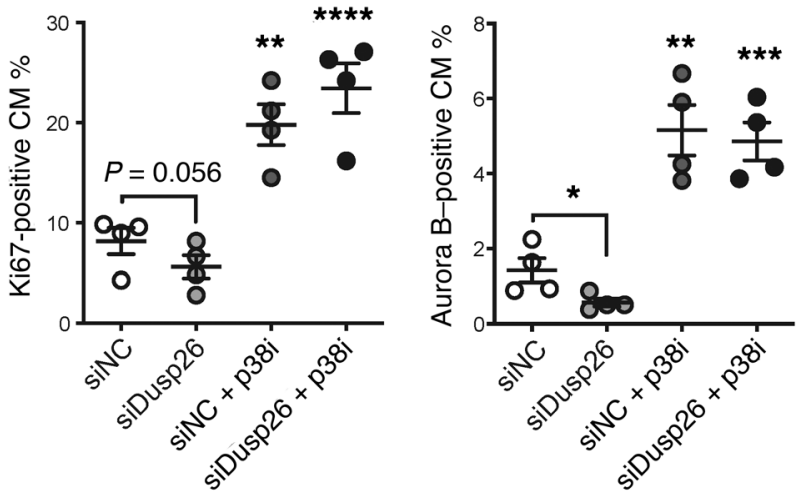

Figure 7. DUSP26 expression upon chamber-specific p38 activation. (A) Chamber-specific DUSP26 mRNA levels in the neonatal mouse heart at P1, $\mathrm{P} 3$, and P7 as indicated $(n=6$; mean $\pm \mathrm{SEM})$. ${ }^{*} P<0.01,{ }^{*} P<0.05$ (LV vs. RV); ${ }^{*} P<0.05$ (control vs. p38-cdKO). (B) p38 activation in cardiomyocytes treated with siRNA against Dusp26 ( $n=3$; mean \pm SEM). (C) mRNA expression of genes differentially expressed in each ventricle in the siDusp26treated cardiomyocytes $(n=6$; mean \pm SEM). ( $(\mathbf{D}$ and $\mathbf{E})$ The levels of Ki67-positive (D) or Aurora B-positive (E) cardiomyocytes (CM) following different treatments with p38 inhibitor (p38i, SB202190) and siDusp26 ( $n=4$; mean $\pm \mathrm{SEM})$. ${ }^{* * *} P<0.0001,{ }^{* * *} P<0.001,{ }^{* *} P<0.01,{ }^{*} P<0.05$ (vs. scrambled siRNA control [siNC]).

interconnected stress-response pathways, p38 MAPK and IRE1 $\alpha$ / $\mathrm{XBP} 1$, in the regulation of chamber-specific cardiomyocyte proliferation during normal perinatal transition. To our knowledge, this is the first cardiomyocyte-intrinsic signaling pathway identified in postnatal chamber-specific remodeling.

Chamber-specific activation of p38 MAPK in the RV during neonatal development is one of the key findings of this study. While p38 MAPK activity was elevated in the RV at P1, P3, and $\mathrm{P} 7$, its activity was diminished below detectable levels in the LV at the same perinatal time points. Despite the dramatic changes in hemodynamics, oxygen, and hormone environment during this transition period, other parallel branches of MAPKs, i.e., ERK and JNK, were not altered. This level of specificity raises question about the involvement of hormones or mechanical stress in the observed differential p38 activation between the 2 chambers, as those external factors often affect all branches of the MAPK pathway (31). This is also consistent with the notion that canonical upstream kinases (MKK3 and MKK6) for p38 do not show differential activation in the perinatal LV versus RV. Although the complete molecular basis for the chamber specificity remains to be determined, it is plausible that a cardiomyocyte-intrinsic signaling network, such as differential expression of DUSP26, contributes to the chamber-specific p38 activation. Further investigation will be needed to determine whether a cardiomyocyte-autonomous mechanism versus environmental factors dictate p38 activation in vivo. In this study, the chamberspecific activation of $\mathrm{p} 38$ was observed in the ventricles; however, their activities and role in left versus right atria will still need to be established.

DUSP26 possesses potent phosphatase activity to dephosphorylate and inactivate p38 MAPK, while has little effect on the other MAPKs, ERK1/2 and JNK (32). Our data indicate that the LV-enriched expression of this protein phosphatase may contribute to the chamber-specific inactivation of p38 MAPK in the LV during postnatal heart development. However, we cannot exclude the possibility that other protein phosphatases or p38 modulators also contribute to RV- versus LV-specific p38 activation in the perinatal heart.

In this study, we provided extensive evidence that RVspecific p38 MAPK activity is a necessary signaling event to regulate cardiomyocyte proliferation, hypertrophy, and apoptosis. However, our understanding of the downstream events responsible for each of these cellular processes is incomplete. Based on the evidence obtained from the p38-cdKO hearts, we conclude that the AKT pathway may not be involved in this process, which contradicts an earlier report linking AKT with p38 activation in neonatal cardiomyocyte proliferation regulation (33). In contrast, we observed an upregulation of several other 


\section{A}

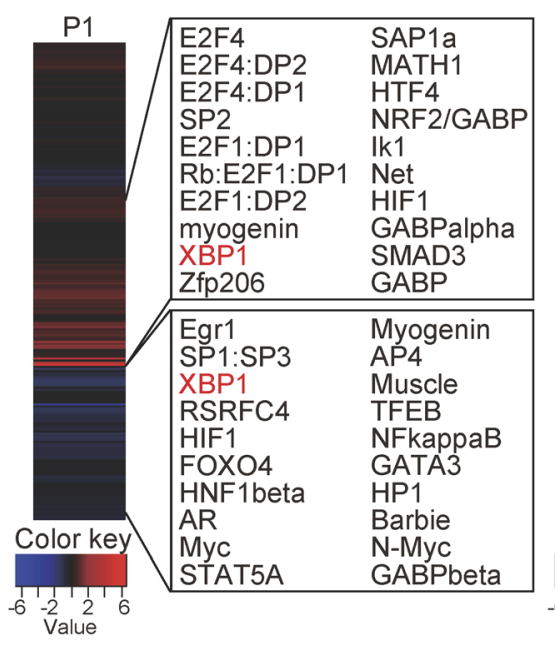

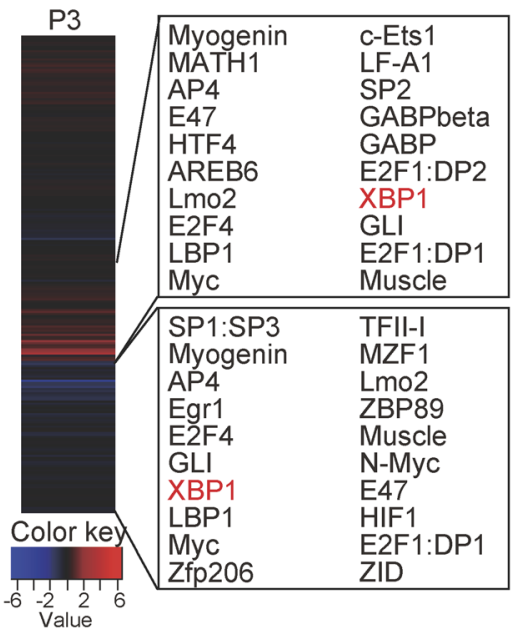
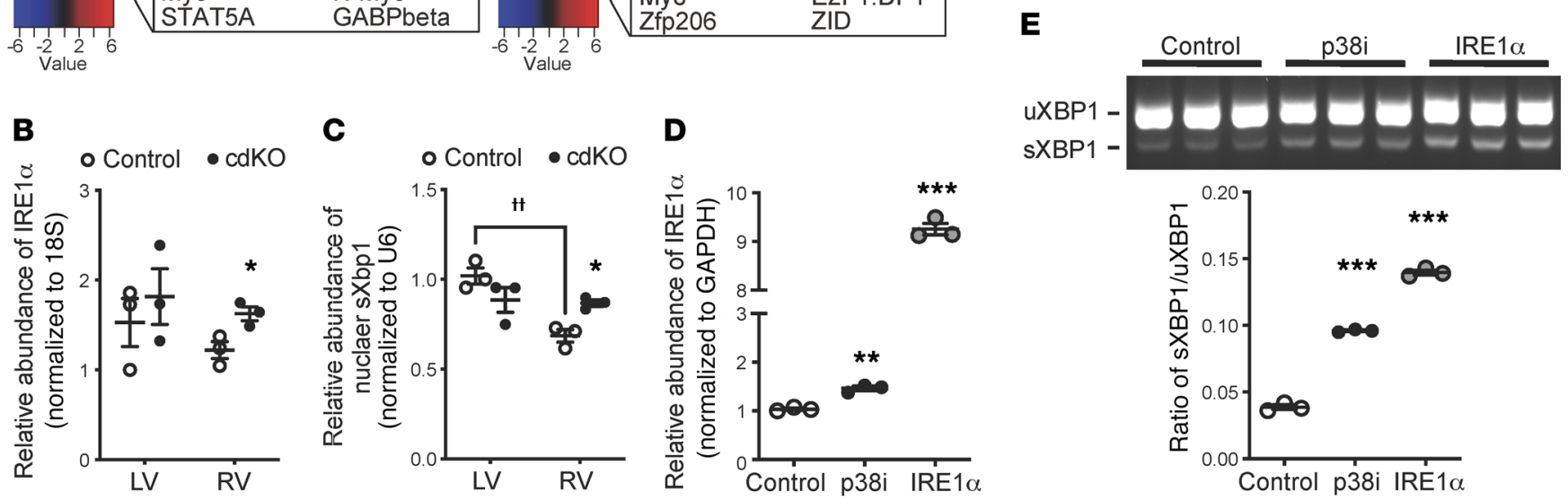

Figure 8. IRE1 $\alpha$ /XBP1 and p38 MAPK activity in cardiomyocytes. (A) Whole-genome rVISTA analysis of the differentially expressed genes in the P1 and P3 p38-cdKO RV vs. the control RV. The top 20 candidate transcription factors are listed for the upregulated genes (upper panels) and the downregulated genes (bottom panels). (B) Chamber-specific expression of IRE1 $\alpha$ mRNA in the P3 control and p38-cdKO ventricles $\left(n=3\right.$; mean \pm SEM). ${ }^{*} P<0.05$ (vs. control). (C) Chamber-specific expression of nuclear sXbp1 mRNA at P3 in the control and the p38-cdkO ventricles $\left(n=3 ;\right.$ mean \pm SEM). ${ }^{*} P<0.05$ (vs. control); ${ }^{t+} P<0.01$ (LV vs. RV). (D) IRE1 $\alpha$ expression in the rat neonatal ventricular myocytes (NRVMs) treated with p38 inhibitor (p38i, SB202190) or Adv-IRE1 $\alpha$ ( $n=$ 3; mean \pm SEM). (E) The unspliced (uXbp1) and spliced (sXbp1) Xbp1 mRNA levels in the NRVMs treated with p38i (SB202190) or Adv-IRE1 $\alpha(n=3 ;$ mean \pm SEM). ${ }^{* *} P<0.001,{ }^{* *} P<0.01$ (vs. control).

growth factors in the p38-cdKO RV, including TGF- $\beta 2$, insulin growth factor, and neuregulin 1 (Nrg1). Each of them, especially Nrg1, is known to play a role in chamber morphogenesis and cardiomyocyte proliferation regulation (34-38). Bmp10 was also upregulated in the p38-cdKO RV. Both Nrg1 and Bmp10 are essential for chamber formation during fetal development (37). Therefore, p38 MAPK may regulate RV-specific cardiomyocyte proliferation and growth via several downstream growth signaling molecules and morphogens. However, their specific contribution to the p38-mediated chamber-specific postnatal growth still needs to be experimentally demonstrated. In addition to the apparent impact on cardiomyocyte proliferation, the in vitro evidence also indicates that p38 activity affects neonatal cardiomyocyte binucleation. Binucleation is a part of the maturation process in neonatal mammalian hearts. It is interesting to note that a recent systems-based study found that the cardiac-specific MAPK Tnni3k is a key regulator of neonatal myocyte ploidy (39). Tnni3k is also an upstream regulator of p38 MAPK involved in cardiac injury and pathological remodeling in the adult heart $(40,41)$. Therefore, the specific contribution of Tnni3K in p38-dependent effects on cardiomyocyte binucleation to $\mathrm{RV}$-specific remodeling remains to be demonstrated in vivo.

Based on unbiased transcriptome profiling, we found that $\mathrm{XBP1}$ is also a potential transcription factor with a significant contribution to $\mathrm{p} 38 \mathrm{MAPK}-$ dependent gene regulation. Indeed, IRE1 $\alpha / \mathrm{sXBP} 1$ was selectively upregulated in the p38-cdKO hearts in vivo as well as in the p38-inactivated NRVMs in vitro. In addition, IRE1 $\alpha$ expression in cardiomyocytes was sufficient to promote cell proliferation, and p38 inhibition-induced NRVM proliferation was blunted by XBP1 inactivation. These results suggest that IRE1 $\alpha / \mathrm{XBP} 1$ signaling is both necessary and sufficient for p38-dependent neonatal cardiomyocyte proliferation. IRE1 $\alpha$ / sXBP1 signaling is also known to regulate proliferation in cancer cells and pancreatic $\beta$ cells (42-44). Furthermore, it has a prosurvival role under various conditions $(45,46)$. Interestingly, a recent report by Wang et al. demonstrated that $\mathrm{SXBP} 1$ has a protective effect under ischemia/reperfusion in the heart through the coupling of the unfolded protein response to the hexosamine biosynthetic pathway (47), suggesting that IRE1 $\alpha / \mathrm{sXBP1}$ could also be involved in the regulation of cardiomyocyte apoptosis in neo- 
A

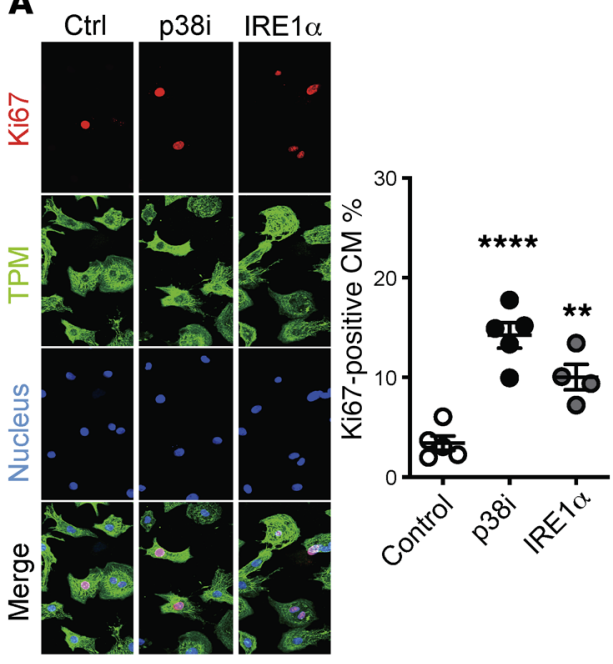

D
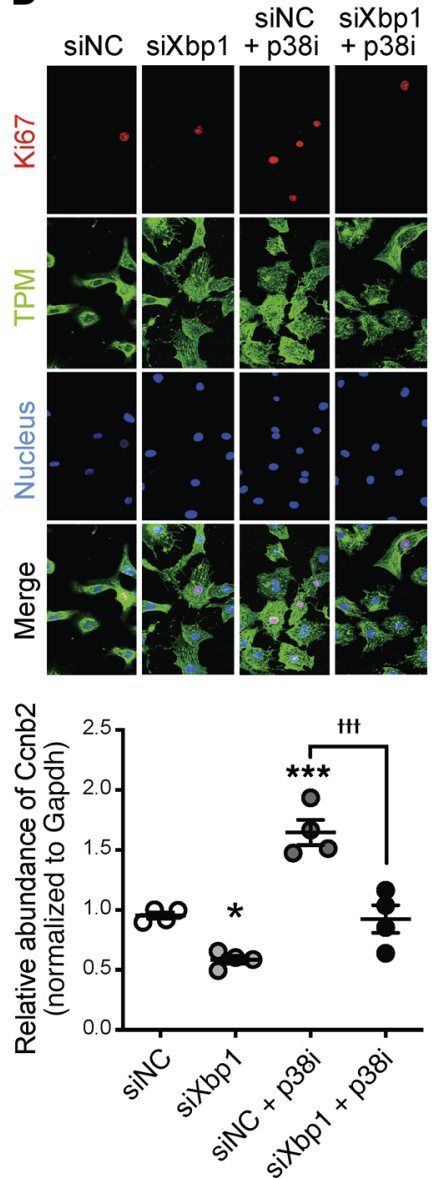

B

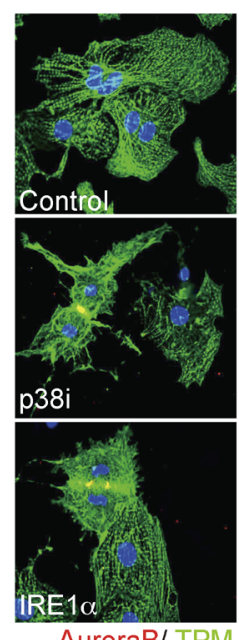

AuroraB/ TPM
C

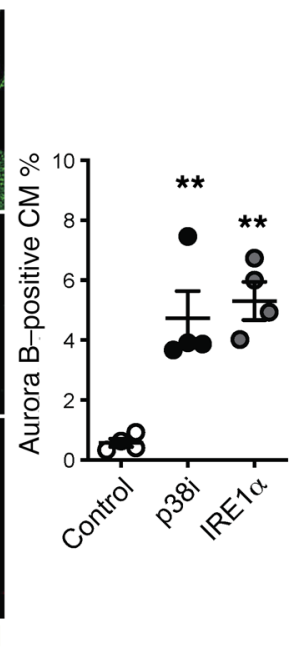

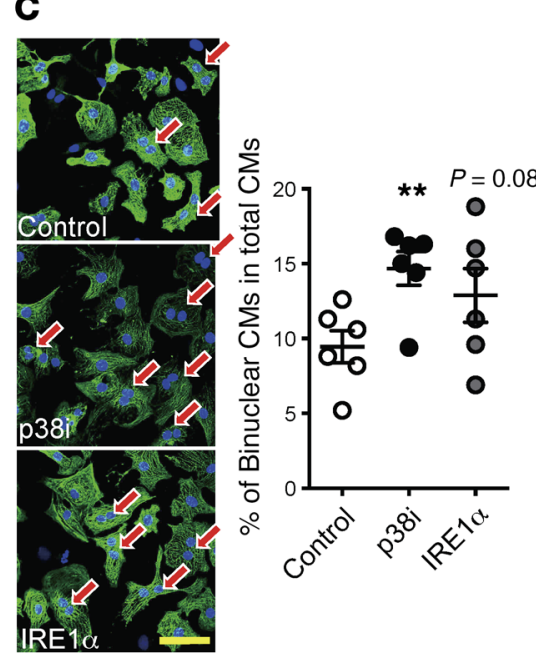

$\mathbf{E}$
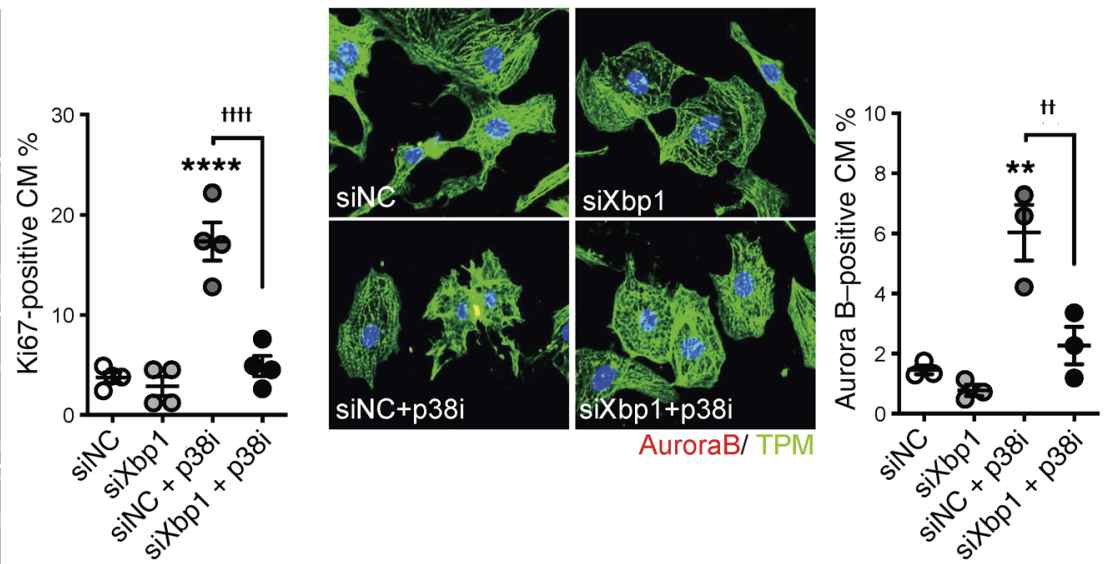

Figure 9. IRE1 $\alpha$ /XBP1 and p38 MAPK activity in cardiomyocyte proliferation. (A) Representative immunohistochemical images and quantification of Ki67-positive, (B) Aurora B-positive, and (C) binucleated NRVMs treated with p38 inhibitor (p38i, SB202190) or Adv-IRE1 $\alpha(n=4-6$; mean \pm SEM). TPM, tropomyosin. (D) Representative immunohistochemistry images/quantification of Ki67-positive and (E) Aurora B-positive NRVMs treated with p38i (SB202190) plus nonspecific (siNC) or Xbp1-specific (siXbp1) silencing ( $n=3-4$; mean \pm SEM). Original magnification, $\times 60$ (A and $\mathbf{D})$ and $\times 120$ (B and E). Scale bar: $50 \mu \mathrm{m}$ (C). (F) mRNA levels of Ccnb2 (encoding cyclin B2) in NRVMs treated with p38i (SB202190) plus nonspecific (siNC) or Xbp1-specific (siXbp1) gene silencing $\left(n=4\right.$; mean \pm SEM). ${ }^{* * * *} P<0.0001,{ }^{* * *} P<$ $0.001,{ }^{* *} P<0.01,{ }^{*} P<0.05$ (vs. siNC); ${ }^{++t+} P<0.0001,{ }^{+t+} P<0.001,{ }^{+\dagger} P<0.01$ (LV vs. RV, siNC + p38i vs. siXbp1 + p38i).

natal hearts. In short, although p38-mediated signaling appears to be essential for all 3 aspects of cellular processes associated with differential development between the LV versus the RV, i.e., cardiomyocyte proliferation, hypertrophy, and apoptosis, the specific downstream mechanisms may be different for each process. Indeed, the molecular basis for p38-dependent regulation of IRE1 $\alpha /$ XBP1 activity remains unclear and should be further elucidated. In addition, because the identification of IRE1 $\alpha / \mathrm{XBP} 1$ as a downstream signaling axis is based largely on in vitro evidence, more studies will be needed to establish its contribution in vivo. In particular, it needs to be further demonstrated whether this pathway is a chamber-specific cardiomyocyte-autonomous signaling pathway or a mediator of yet-to-be identified external factor(s) that dictate chamber-specific remodeling in the postnatal heart.

Chamber-specific remodeling is critical to postnatal cardiac physiology. It is intriguing to note that loss of p38-mediated sig- 
naling in neonatal cardiomyocytes led to approximately $25 \%$ mortality in neonates. However, despite a significant elevation of pulmonary pressure due to enlarged RVs, the surviving $\mathrm{p} 38$-cdKO mice appeared to remain viable, with no signs of LV failure up to 8 months. The longer-term impact of such abnormality on cardiac output and viability will still need to be investigated. Nevertheless, the physiological role of $\mathrm{p} 38 / \mathrm{IRE} 1 \alpha / \mathrm{XBP} 1$ signaling in RVspecific postnatal development uncovered in this study may also have implications in the pathogenesis of congenital heart disease where genetic predisposition and postnatal pathological stress may interfere with normal cardiomyocyte proliferation, growth, and survival in a chamber-specific fashion.

\section{Methods}

Supplemental materials, including Supplemental Methods, 6 Supplemental Figures, 8 Supplemental Tables, 4 Supplemental Videos, and complete unedited blots can be found online.

The raw data, analytic methods, and study materials are described in full as online supplemental information. The genetically engineered mice and expression plasmids are available from Yibin Wang's lab at UCLA. The list of the differentially expressed genes are included in the supplemental material (Supplemental Tables 2-7). The entire RNA-seq data set has been deposited at NIH-SRA under accession number PRJNA639143. Information for antibodies used in this report is listed in Supplemental Table 8.

Animals. The cardiomyocyte-specific p38 MAPK $\alpha$ (Mapk14) and $\beta$ (Mapk11) knockout mice were generated by crossing Mapk14 loxP and Mapk11 loxP mice with myosin light chain 2a-Cre (Mlc2a-cre) mice as previously reported $(18,33)$. The $M l c 2 a$-cre mice have the cre cDNA knocked in within the $M l c 2 a$ locus and develop no cardiac phenotype, as reported in several earlier studies $(18,48,49)$. Although endogenous $M l c 2 a$ gene expression is restricted to the atrial cardiomyocytes in the adult heart, its expression is distributed in all chambers of the embryonic hearts, leading to efficient p38 KO in all ventricular cardiomyocytes. Age- and sex-matched littermates with homozygous
Mapk14 loxP and Mapk11 loxP alleles but no Mlc2a-cre allele were used as the controls in this study.

Statistics. Data are indicated as mean \pm SEM. Statistical analysis was performed using an unpaired, 2-tailed Student's $t$ test between 2 groups or 1-way ANOVA with multiple comparisons test (Tukey) among multiple groups. $P$ values less than 0.05 were considered statistically significant.

Study approval. All animal handling and procedures were carried out in compliance with UCLA guidelines and approved by UCLA IACUC.

\section{Author contributions}

TY and YW designed all experiments. TY, JH, and JL performed all molecular and animal experiments, data analysis, data interpretation, and manuscript preparation. YW managed funding and contributed experimental design, data interpretation, and manuscript preparation. QZ, TC, CR, MT, and XX contributed RNA-seq data analysis. YD, KS, TH, and RK contributed the light-sheet imaging. ZX and SR contributed animal analysis. SM contributed data interpretation and manuscript preparation.

\section{Acknowledgments}

This work was supported in part by grants from the US NIH (HL070079, HL103205, HL108186, and HL110667) and DoD/ DOD/DHP PRMRP-IIRA (PR171540) to YW. TY was supported by the Construction of Lab-exchange Type Health \& Biomedical Science Research Consortium, Japan. The authors wish to thank Haiying Pu for excellent technical assistance with this study.

Address correspondence to: Yibin Wang, Department of Anesthesiology and Division of Molecular Medicine, Room BH 569 CHS, 650 Charles E. Young Drive, Box 957115, David Geffen School of Medicine, UCLA, Los Angeles, California 90095, USA. Phone: 310.206.5197; Email: yibinwang@ mednet.ucla.edu.
1. Gill AW. Postnatal cardiovascular adaptation. Arch Dis Child Fetal Neonatal Ed. 2019;104(2):F220-F224.

2. Kelly RG. The second heart field. Curr Top Dev Biol. 2012;100:33-65.

3. Fernandez E, Siddiquee Z, Shohet RV. Apoptosis and proliferation in the neonatal murine heart. Dev Dyn. 2001;221(3):302-310.

4. Smolich JJ, Walker AM, Campbell GR, Adamson TM. Left and right ventricular myocardial morphometry in fetal, neonatal, and adult sheep. Am J Physiol. 1989;257(1 pt 2):H1-H9.

5. Bishop SP. The myocardial cell: normal growth, cardiac hypertrophy and response to injury. Toxicol Pathol. 1990;18(4 pt 1):438-453.

6. Tucker DC, Bishop SP. Use of embryonic heart grafted in oculo to assess neurohumoral controls of cardiac development. Toxicol Pathol. 1990;18(4 pt 1):531-540.

7. Kim EK, Choi EJ. Pathological roles of MAPK signaling pathways in human diseases. Biochim Biophys Acta . 2010;1802(4):396-405.

8. Kyriakis JM, Avruch J. Mammalian mitogenactivated protein kinase signal transduction pathways activated by stress and inflammation. Physiol Rev. 2001;81(2):807-869.

9. Lee JC, Kumar S, Griswold DE, Underwood DC, Votta BJ, Adams JL. Inhibition of p38 MAP kinase as a therapeutic strategy. Immunopharmacology. 2000;47(2-3):185-201.

10. Rodríguez-Carballo E, Gámez B, Ventura F. p38 MAPK signaling in osteoblast differentiation. Front Cell Dev Biol. 2016;4:40.

11. Efimova T. p38delta mitogen-activated protein kinase regulates skin homeostasis and tumorigenesis. Cell Cycle. 2010;9(3):498-405.

12. Keren A, Tamir Y, Bengal E. The p38 MAPK signaling pathway: a major regulator of skeletal muscle development. Mol Cell Endocrinol. 2006;252(1-2):224-230.

13. Yokota T, Wang Y. p38 MAP kinases in the heart. Gene. 2016;575(2 pt 2):369-376.

14. Denise Martin E, De Nicola GF, Marber MS. New therapeutic targets in cardiology: p38 alpha mitogen-activated protein kinase for ischemic heart disease. Circulation. 2012;126(3):357-368.

15. Marber MS, Molkentin JD, Force T. Developing small molecules to inhibit kinases unkind to the heart: p38 MAPK as a case in point. Drug Discov Today Dis Mech. 2010;7(2):e123-e127.

16. Nishida K, et al. p38alpha mitogen-activated protein kinase plays a critical role in cardiomyocyte survival but not in cardiac hypertrophic growth in response to pressure overload. Mol Cell Biol. 2004;24(24):10611-10620.

17. Rose BA, et al. Cardiac myocyte p $38 \alpha$ kinase regulates angiogenesis via myocyte-endothelial cell cross-talk during stress-induced remodeling in the heart. J Biol Chem. 2017;292(31):12787-12800.

18. Peng X, et al. Cardiac developmental defects and eccentric right ventricular hypertrophy in cardiomyocyte focal adhesion kinase (FAK) conditional knockout mice. Proc Natl Acad Sci U S A. 2008;105(18):6638-6643.

19. Xin M, et al. Hippo pathway effector Yap promotes cardiac regeneration. Proc Natl Acad Sci U S A. 2013;110(34):13839-13844.

20. Lin Z, Pu WT. Releasing YAP from an $\alpha$-catenin trap increases cardiomyocyte proliferation. Circ Res. 2015;116(1):9-11.

21. Morikawa Y, Heallen T, Leach J, Xiao Y, Martin JF. Dystrophin-glycoprotein complex sequesters Yap 
to inhibit cardiomyocyte proliferation. Nature. 2017;547(7662):227-231.

22. Kerkela R, et al. Deletion of GSK-3beta in mice leads to hypertrophic cardiomyopathy secondary to cardiomyoblast hyperproliferation. J Clin Invest. 2008;118(11):3609-3618.

23. Calfon M, et al. IRE1 couples endoplasmic reticulum load to secretory capacity by processing the XBP-1 mRNA. Nature. 2002;415(6867):92-96.

24. Yoshida H, Oku M, Suzuki M, Mori K. pXBP1(U) encoded in XBP1 pre-mRNA negatively regulates unfolded protein response activator $\mathrm{pXBP1(S)}$ in mammalian ER stress response. J Cell Biol. 2006;172(4):565-575.

25. Yoshida H, Matsui T, Yamamoto A, Okada T, Mori K. XBP1 mRNA is induced by ATF 6 and spliced by IRE1 in response to ER stress to produce a highly active transcription factor. Cell. 2001;107(7):881-891.

26. Walsh S, Pontén A, Fleischmann BK, Jovinge S. Cardiomyocyte cell cycle control and growth estimation in vivo--an analysis based on cardiomyocyte nuclei. Cardiovasc Res. 2010;86(3):365-373.

27. Leone M, Magadum A, Engel FB. Cardiomyocyte proliferation in cardiac development and regeneration: a guide to methodologies and interpretations. Am J Physiol Heart Circ Physiol. 2015;309(8):H1237-H1250.

28. Matsuyama D, Kawahara K. Oxidative stressinduced formation of a positive-feedback loop for the sustained activation of $\mathrm{p} 38$ MAPK leading to the loss of cell division in cardiomyocytes soon after birth. Basic Res Cardiol. 2011;106(5):815-828.

29. Shozawa T, Okada E, Kawamura K, Sageshima M, Masuda H. Development of binucleated myocytes in normal and hypertrophied human hearts. Am J Cardiovasc Pathol. 1990;3(1):27-36.

30. Soonpaa MH, Kim KK, Pajak L, Franklin M, Field LJ. Cardiomyocyte DNA synthesis and binucleation during murine development. Am J Physiol.
1996;271(5 pt 2):H2183-H2189.

31. Rose BA, Force T, Wang Y. Mitogen-activated protein kinase signaling in the heart: angels versus demons in a heart-breaking tale. Physiol Rev. 2010;90(4):1507-1546.

32. Yu W, Imoto I, Inoue J, Onda M, Emi M, Inazawa J. A novel amplification target, DUSP26, promotes anaplastic thyroid cancer cell growth by inhibiting p38 MAPK activity. Oncogene. 2007;26(8):1178-1187.

33. Engel FB, et al. p38 MAP kinase inhibition enables proliferation of adult mammalian cardiomyocytes. Genes Dev. 2005;19(10):1175-1187.

34. Gemberling M, Karra R, Dickson AL, Poss KD. $\mathrm{Nrg} 1$ is an injury-induced cardiomyocyte mitogen for the endogenous heart regeneration program in zebrafish. Elife. 2015;4:e05871.

35. Baliga RR, et al. NRG-1-induced cardiomyocyte hypertrophy. Role of PI-3-kinase, p70(S6K), and MEK-MAPK-RSK. Am J Physiol. 1999;277(5):H2O26-H2O37.

36. Hertig CM, Kubalak SW, Wang Y, Chien KR. Synergistic roles of neuregulin- 1 and insulinlike growth factor-I in activation of the phosphatidylinositol 3-kinase pathway and cardiac chamber morphogenesis. J Biol Chem. 1999;274(52):37362-37369.

37. Grego-Bessa J, et al. Notch signaling is essential for ventricular chamber development. Dev Cell. 2007;12(3):415-429.

38. D'Uva G, et al. ERBB2 triggers mammalian heart regeneration by promoting cardiomyocyte dedifferentiation and proliferation. Nat Cell Biol. 2015;17(5):627-638.

39. Patterson M, et al. Frequency of mononuclear diploid cardiomyocytes underlies natural variation in heart regeneration. Nat Genet. 2017;49(9):1346-1353.

40. Lai ZF, et al. Overexpression of TNNI3K, a cardiac-specific MAP kinase, promotes P19CL6- derived cardiac myogenesis and prevents myocardial infarction-induced injury. Am J Physiol Heart Circ Physiol. 2008;295(2):H708-H716.

41. Vagnozzi RJ, et al. Inhibition of the cardiomyocyte-specific kinase TNNI3K limits oxidative stress, injury, and adverse remodeling in the ischemic heart. Sci Transl Med. 2013;5(207):207ra141.

42. Thorpe JA, Schwarze SR. IRE1alpha controls cyclin A1 expression and promotes cell proliferation through XBP-1. Cell Stress Chaperones. 2010;15(5):497-508.

43. Romero-Ramirez L, et al. XBP1 is essential for survival under hypoxic conditions and is required for tumor growth. Cancer Res. 2004;64(17):5943-5947.

44. Xu T, et al. The IRE1 $\alpha$-XBP1 pathway regulates metabolic stress-induced compensatory proliferation of pancreatic $\beta$-cells. Cell Res. 2014;24(9):1137-1140.

45. Iwakoshi NN, Lee AH, Vallabhajosyula P, Otipoby KL, Rajewsky K, Glimcher LH. Plasma cell differentiation and the unfolded protein response intersect at the transcription factor XBP-1. Nat Immunol. 2003;4(4):321-329.

46. Casas-Tinto S, Zhang Y, Sanchez-Garcia J, Gomez-Velazquez M, Rincon-Limas DE, Fernandez-Funez P. The ER stress factor XBP1s prevents amyloid-beta neurotoxicity. Hum Mol Genet. 2011;20(11):2144-2160.

47. Wang ZV, et al. Spliced X-box binding protein 1 couples the unfolded protein response to hexosamine biosynthetic pathway. Cell. 2014;156(6):1179-1192.

48. Wettschureck N, et al. Absence of pressure overload induced myocardial hypertrophy after conditional inactivation of Galphaq/Galpha11 in cardiomyocytes. Nat Med. 2001;7(11):1236-1240.

49. Clay H, et al. Sphingosine 1-phosphate receptor-1 in cardiomyocytes is required for normal cardiac development. Dev Biol. 2016;418(1):157-165. 